\title{
11 Schlussbetrachtung der empirischen Untersuchung
}

Im vorliegenden Kapitel werden für die empirischen Erkenntnisse aus den vorherigen Kapiteln Robustheitstests und ergänzende Analysen durchgeführt, die Grenzen der Untersuchung aufgezeigt, deren Ergebnisse kritisch gewürdigt, die Forschungsfragen beantwortet, der weitere Forschungsbedarf umrissen sowie die Arbeit zusammengefasst.

\subsection{Robustheitstests}

Vereinfacht gesagt wird im vorliegenden Abschnitt untersucht, ob die bisherigen empirischen Erkenntnisse bei kleineren Änderungen am ökonometrischen Modell beibehalten werden können. ${ }^{1581}$ Diese Robustheitstests sollen zeigen, ob die bisherigen empirischen Erkenntnisse durch die verwendeten Messverfahren systematisch begünstigt wurden. Falls die bisherigen empirischen Erkenntnisse trotz den veränderten Messverfahren im Wesentlichen beibehalten werden können, scheinen sie über eine gewisse Robustheit zu verfügen. Falls die bisherigen empirischen Erkenntnisse bei veränderten Messverfahren dagegen im Wesentlichen ungültig werden, gelten sie als sensitiv und sollten besonders vorsichtig interpretiert werden. Im Folgenden werden vier Robustheitstests durchgeführt:

(1) Vergleichbare Studien nehmen teilweise ein sog. Winsorizing vor, mit denen die Werte unter- und oberhalb von bestimmten Quantilen von verhältnisskalierten Variablen auf die jeweiligen Quantilwerte festgelegt werden. ${ }^{1582}$ Das Winsorizing sollen verhindern, dass einzelne Extremwerte die Testergebnisse übermäßig beeinflussen. Hierfür wurde für alle verhältnisskalierten Variablen ein Winsorizing zu den 1\%- und 99 \%-Quantilen vorgenommen. ${ }^{1583}$ Die Testergebnisse blieben durch das Winsorizing inhaltlich unverändert und werden nicht abgedruckt.

(2) Die Regressionsgleichungen (18a) und (18b), mit denen die notwendige und die hinreichende Bedingung für abschlusspolitische Ergebnisspaltungen bei ineffizienten Größen der fortgeführten Geschäftsbereiche getestet wurden, enthalten $B M_{-} G 1_{i, t}$ als Interaktionsvariable und $B M_{i, t}$ als Kontrollvariable. Die verhältnisskalierte Kontrollvariable $B M_{i, t}$ misst die Verhältnisse der Buch- zu den Marktwerten des Eigenkapitals. Der nominalskalierten Interaktionsvariable $B M_{-} G 1_{i, t}$ wird der Wert 1 zugewiesen, falls der Buch- über dem Marktwert des Eigenkapitals liegt. Andernfalls wird

1581 Hierzu Wooldridge, J. M. (2020), S. 317.

1582 Siehe Ji, Y./Potepa, J./Rozenbaum, O. (2019), S. 9; Skousen, C./Sun, L./Wu, K. (2019), S. 119. Dagegen verzichten Anthonius/Murwaningsari, E. (2018), S. 108-116; Barua, A./Lin, S./Sbaraglia, A. M. (2010), S. 1485-1509; Chagnaadorj, O. (2018), S. 1-88; Curtis, A./McVay, S./Wolfe, M. (2014), S. 190-201; Darrough, M./Lee, Y. G./Oh, H. I. (2019), S. 185-206; Seve, F. (2016), S. 130 auf ein Winsorizing.

1583 Die Werte unterhalb der 1\%-Quantile werden somit auf die 1\%-Quantile und die Werte oberhalb der 99 \%-Quantile auf die $99 \%$-Quantil festgelegt. Ebenso Ji, Y./Potepa, J./Rozenbaum, O. (2019), S. 9; Skousen, C./Sun, L./Wu, K. (2019), S. 119.

(C) Der/die Autor(en) 2021

K. Czupalla, Abschlusspolitische Ergebnisspaltungen mit

aufgegebenen Geschäftsbereichen nach IFRS 5, Auditing and

Accounting Studies, https://doi.org/10.1007/978-3-658-34436-8_11 
$B M \_G 1_{i, t}$ mit dem Wert 0 kodiert. Da $B M_{i, t}$ die Verhältnisse der Buch- zu den Marktwerten des Eigenkapitals entspricht und $B M_{-} G 1_{i, t}$ mittelbar von diesem Verhältnissen abhängt, ist zwischen $B M_{i, t}$ und $B M_{-} G 1_{i, t}$ eine starke Korrelation zu erwarten. Eine starke Korrelation zwischen zwei unabhängigen Variablen in einer Regressionsgleichung wird Multikollinearität genannt und kann die Interpretation der partiellen Effekte von einzelnen unabhängigen Variablen auf die abhängige Variable erschweren. ${ }^{1584}$ In den Regressionsgleichungen (18a), (18b), (25a) und (25b) könnten sich die Zusammenhänge zwischen $B M_{i, t}$ und der abhängigen Variable sowie zwischen $B M_{-} G 1_{i, t}$ und der abhängigen Variable aufgrund der Multikollinearität überlagern. ${ }^{1585}$ Daher wurden alle Regressionsgleichungen, die $B M_{-} G 1_{i, t}$ als Interaktions- und $B M_{i, t}$ als Kontrollvariable enthalten, ohne $B M_{i, t}$ geschätzt. Die Testergebnisse blieben inhaltlich unverändert und werden nicht abgedruckt.

(3) Abschlusspolitische Ergebnisspaltungen werden in der vorliegenden Arbeit mit den Zusammenhängen zwischen einerseits den Ergebnissen aus den aufgegebenen Geschäftsbereichen und andererseits der unerwarteten bereinigten Betriebsergebnismargen bzw. den unerwarteten Differenzen der bereinigten Betriebsergebnismargen gemessen. Die unerwarteten bereinigten Betriebsergebnismargen wurden als $U E W_{-} B E_{F, i, t}=B E_{F, i, t}-\mathbb{E}\left[B E_{F, i, t}\right]$ und die unerwarteten Differenzen der bereinigten Betriebsergebnismargen als $U E W_{-} \Delta B E_{F, i, t+1}=\Delta B E_{F, i, t+1}-\mathbb{E}\left[\Delta B E_{F, i, t+1}\right]$ definiert. ${ }^{1586}$ Im Einklang mit McVay (2006) und Barua/Lin/Sbaraglia (2010) wurden die $U E W_{-} \triangle B E_{F, i, t+1}$ empirisch vollständig getrennt von den $U E W_{-} B E_{F, i, t}$ berechnet, ${ }^{1587}$ obwohl die Umformungen in der Gleichung (32) zeigen, ${ }^{1588}$ dass $U E W_{-} B E_{F, i, t}$ in der Theorie ein Bestandteil von $U E W \_\Delta B E_{F, i, t+1}$ ist. Daher werden alle Regressionen, welche die $U E W \_\Delta B E_{F, i, t+1}$ als abhängige Variable enthalten, ${ }^{1589}$ nachfolgend erneut durchgeführt, wobei die $U E W_{-} \triangle B E_{F, i, t+1}$ ohne separate Schätzungen $^{1590}$ als $U E W_{-} \Delta B E_{F, i, t+1}=U E W_{-} B E_{F, i, t+1}-U E W_{-} B E_{F, i, t}$ berechnet werden.

1584 Siehe Wooldridge, J. M. (2020), S. 89-91/134/144.

1585 In den Regressionsgleichungen (18a) und (25a) bildet $U E W_{-} B E_{F, i, t}$ die abhängige Variable, während die abhängige Variable in den Regressionsgleichungen (18b) und (25b) $U E W \_\Delta B E_{F, i, t+1}$ lautet.

1586 Siehe für $U E W_{-} B E_{F, i, t}$ die Gleichung (9) und für $U E W \_\Delta B E_{F, i, t+1}$ die Gleichung (14).

1587 Siehe McVay, S. E. (2006), S. 509/511/514/516 und später Barua, A./Lin, S./Sbaraglia, A. M. (2010), S. $1494 \mathrm{f} . / 1499$.

1588 Vgl. zu Summen und Differenzen von Erwartungswerten Kosfeld, R./Eckey, H.-F./Türck, M. (2019), S. 353-356.

1589 Die abhängige Variable $U E W \_\Delta B E_{F, i, t+1}$ wird in den hinreichenden Bedingungen für die Existenz von abschlusspolitischen Ergebnisspaltungen und in den zweiten Möglichkeiten bzw. hinreichenden Bedingungen für die Prävention von abschlusspolitischen Ergebnisspaltungen verwendet.

1590 Siehe für die ursprüngliche Schätzung von $U E W_{-} \Delta B E_{F, i, t+1}$ die Gleichungen (10), (13) und (14). 


$$
\begin{aligned}
U E W_{-} \Delta B E_{F, i, t+1} & =\Delta B E_{F, i, t+1}-\mathbb{E}\left[\Delta B E_{F, i, t+1}\right] \\
& =B E_{F, i, t+1}-B E_{F, i, t}-\mathbb{E}\left[B E_{F, i, t+1}-B E_{F, i, t}\right] \\
& =B E_{F, i, t+1}-B E_{F, i, t}-\left(\mathbb{E}\left[B E_{F, i, t+1}\right]-\mathbb{E}\left[B E_{F, i, t}\right]\right) \\
& =B E_{F, i, t+1}-B E_{F, i, t}-\mathbb{E}\left[B E_{F, i, t+1}\right]+\mathbb{E}\left[B E_{F, i, t}\right] \\
& =B E_{F, i, t+1}-\mathbb{E}\left[B E_{F, i, t+1}\right]-B E_{F, i, t}+\mathbb{E}\left[B E_{F, i, t}\right] \\
& =B E_{F, i, t+1}-\mathbb{E}\left[B E_{F, i, t+1}\right]-\left(B E_{F, i, t}-\mathbb{E}\left[B E_{F, i, t}\right]\right) \\
& =U E W_{-} B E_{F, i, t+1}-U E W_{-} B E_{F, i, t}
\end{aligned}
$$

In der Tab. 42 sind die Testergebnisse mit der bisherigen und der alternativen Berechnung von $U E W \_\triangle B E_{F, i, t+1}$ zusammengefasst. Bezüglich der Existenz und Prävention von abschlusspolitischen Ergebnisspaltungen bei ineffizienten Unternehmensgrößen bleiben die Testergebnisse unverändert. Dagegen sind mit der alternativen Berechnung von $U E W \_\triangle B E_{F, i, t+1}$ die notwendige und die hinreichende Bedingung für abschlusspolitische Ergebnisspaltungen zum Erhöhen der Ergebnisse aus den fortgeführten Geschäftsbereichen i. S. d. Alternativhypothese $\mathrm{H}_{1.1}$ und zum Erreichen von

\begin{tabular}{|c|c|c|c|c|}
\hline Alternativhypothese & \multicolumn{2}{|c|}{ Bish. $U E W_{-} \Delta B E_{F, i, t+1}$} & \multicolumn{2}{|c|}{ Alt. $U E W_{-} \Delta B E_{F, i, t+1}$} \\
\hline $\begin{array}{l}\text { Existenz von abschlusspolitischen Er- } \\
\text { gebnisspaltungen }\end{array}$ & Notw. Bed. & Hinr. Bed. & Notw. Bed. & Hinr. Bed. \\
\hline $\mathrm{H}_{1.1}$ [Erhöhen fortgeführte Erg.] & $\checkmark$ & $x$ & $\checkmark$ & $\checkmark$ \\
\hline $\mathrm{H}_{1.2}$ [Ineffiz. Unternehmensgrößen] & $\checkmark$ & $\checkmark$ & $\checkmark$ & $\checkmark$ \\
\hline $\mathrm{H}_{1.3}$ [Referenzwerte erreichen] & $\checkmark$ & $x$ & $\checkmark$ & $\checkmark$ \\
\hline $\begin{array}{l}\text { Prävention beim Erhöhen der Ergeb- } \\
\text { nisse aus den fortgeführten Geschäfts- } \\
\text { bereichen }\end{array}$ & 1. Mgl. & 2. $\mathrm{Mgl}$. & 1. Mgl. & 2. Mgl. \\
\hline $\mathrm{H}_{2.1}$ [Aufsichtsräte] & & & $x$ & $x$ \\
\hline $\mathrm{H}_{2.2}$ [Vorstandsvergütungen] & & & $x$ & $x$ \\
\hline $\mathrm{H}_{2.3}$ [Eigentümerstrukturen] & & & $x$ & $x$ \\
\hline $\mathrm{H}_{2.4}$ [Kapitalstrukturen] & & & $x$ & $x$ \\
\hline $\mathrm{H}_{2.5}$ [Prüfungshonorare] & & & $x$ & $x$ \\
\hline $\mathrm{H}_{2.6}$ [Nichtprüfungshonorare] & & & $x$ & $x$ \\
\hline
\end{tabular}
Referenzwerten i. S. d. Alternativhypothese $\mathrm{H}_{1.3}$ nun erfüllt. Die Alternativhypothesen bezüglich der Existenz von abschlusspolitischen Ergebnisspaltungen bei Cookie Jars und Big Baths sind in der Tab. 42 nicht aufgeführt, da bei ihnen bereits die notwendige Bedingung nicht erfüllt war und die alternative Berechnung von $U E W \_\Delta B E_{F, i, t+1}$ nur die Testergebnisse für die hinreichende Bedingung, aber nicht für die notwendige Bedingung, beeinflusst. 


\begin{tabular}{|c|c|c|c|c|}
\hline Alternativhypothese & \multicolumn{2}{|c|}{ Bish. $U E W_{-} \Delta B E_{F, i, t+1}$} & \multicolumn{2}{|c|}{ Alt. $U E W_{-} \Delta B E_{F, i, t+1}$} \\
\hline $\begin{array}{l}\text { Etwaige Pravention beim Erhohen der } \\
\text { Ergebnisse aus den fortgeführten Ge- } \\
\text { schäftsbereichen }\end{array}$ & 1. Bed. & 2. Bed. & 1. Bed. & 2. Bed. \\
\hline $\mathrm{H}_{2.7}[$ Größen AP] & & & $\mathfrak{J}^{*}$ & $\mathfrak{S}^{*}$ \\
\hline $\mathrm{H}_{2.8}[$ Branchenspezialisierungen AP] & & & $\mathfrak{V}^{*}$ & $\mathfrak{J}^{*}$ \\
\hline $\begin{array}{l}\text { Prävention bei ineffizienten Unter- } \\
\text { nehmensgrößen }\end{array}$ & 1. Mgl. & 2. Mgl. & 1. Mgl. & 2. Mgl. \\
\hline $\mathrm{H}_{2.1}$ [Aufsichtsräte] & $x$ & $x$ & $x$ & $x$ \\
\hline $\mathrm{H}_{2.2}$ [Vorstandsvergütungen] & $\checkmark$ & & $\checkmark$ & \\
\hline $\mathrm{H}_{2.3}$ [Eigentümerstrukturen] & $x$ & $x$ & $x$ & $x$ \\
\hline $\mathrm{H}_{2.4}[$ Kapitalstrukturen] & $\checkmark$ & & $\checkmark$ & \\
\hline $\mathrm{H}_{2.5}$ [Prüfungshonorare] & $x$ & $x$ & $x$ & $x$ \\
\hline $\mathrm{H}_{2.6}$ [Nichtprüfungshonorare] & $\checkmark$ & & $\checkmark$ & \\
\hline $\begin{array}{l}\text { Etwaige Prävention bei ineffizienten } \\
\text { Unternehmensgrößen }\end{array}$ & 1. Bed. & 2. Bed. & 1. Bed. & 2. Bed. \\
\hline $\mathrm{H}_{2.7}$ [Größen AP] & $\mathfrak{S}^{*}$ & $\mathfrak{S}^{*}$ & $\mathfrak{J}^{*}$ & $\mathfrak{S}^{*}$ \\
\hline $\mathrm{H}_{2.8}$ [Branchenspezialisierungen AP] & $x^{*}$ & & $x^{*}$ & \\
\hline
\end{tabular}

Prävention beim Erreichen von nichtnegativen ordentlichen Betriebsergebnissen als Referenzwerte

1. Mgl.

2. Mgl.

1. $\mathrm{Mgl}$.

2. Mgl.

$\mathrm{H}_{2.1}$ [Aufsichtsräte]
$\mathrm{H}_{2.2}$ [Vorstandsvergütungen]
$\mathrm{H}_{2.3}$ [Eigentümerstrukturen]
$\mathrm{H}_{2.4}$ [Kapitalstrukturen]
$\mathrm{H}_{2.5}$ [Prüfungshonorare]
$\mathrm{H}_{2.6}$ [Nichtprüfunghonorare]

Etwaige Prävention beim Erreichen von nichtnegativen ordentlichen Betriebsergebnissen als Referenzwerte

$\mathrm{H}_{2.7}$ [Größen AP]

1. Bed.

2. Bed.

1. Bed.

2. Bed.

$\mathrm{H}_{2.8}$ [Branchenspezialisierungen AP]

?
$?$

$X^{*}$

$\checkmark$ : Bed. bzw. Mgl. erfüllt. $\boldsymbol{X}$ : Bed. bzw. Mgl. nicht erfüllt. ?: Bed. bzw. Mgl. wegen Multikollinearität ohne Ergebnis.

* Mit $\mathrm{H}_{2.7}$ und $\mathrm{H}_{2.8}$ wurden keine Wirkungen der Größen respektive Branchenspezialisierungen der Abschlussprüfer auf abschlusspolitische Ergebnisspaltungen postuliert. Daher bedeuten die $\checkmark$ bei $\mathrm{H}_{2.7}$, dass sich die Größen der Abschlussprüfer nicht auf abschlusspolitische Ergebnisspaltungen auswirken. Dagegen signalisiert das $\boldsymbol{X}$ bei $\mathrm{H}_{2.8}$, dass die Branchenspezialisierungen der Abschlussprüfer präventiv wirken.

Tab. 42: Ergebnisse der Hypothesentests zur Prävention von abschlusspolitischen Ergebnisspaltungen mit bisheriger und alternativer abhängiger Variable 
Die Schätzungen der hinreichenden Bedingung für $\mathrm{H}_{1.1}, \mathrm{H}_{1.2}$ und $\mathrm{H}_{1.3}$ können auszugsweise der Tab. 43, der Tab. 44 respektive der Tab. 45 entnommen werden, wobei jeweils die Testergebnisse mit der bisherigen und der alternativen Definition von $U E W \_\triangle B E_{F, i, t+1}$ wiedergegeben sind. In der Tab. 45 sind für vier Referenzwerte, für welche die notwendige Bedingung für $\mathrm{H}_{1.3}$ erfüllt sind, ${ }^{1591}$ aufgrund der alternativen Berechnung von $U E W \_\Delta B E_{F, i, t+1}$ nun auch die hinreichenden Bedingung erfüllt. Konkret können abschlusspolitische Ergebnisspaltungen nun auch beim Erreichen von nichtnegativen ordentlichen Betriebsergebnissen $B E_{-} N N_{F, i, t}$, beim Erreichen von ordentlichen Vorjahresbetriebsergebnissen $B E_{-} M V J_{F, i, t}$, beim Erreichen von Vorjahresergebnissen aus den fortgeführten Geschäftsbereichen $E F G B_{-} M V J_{F, i, t}$ und beim Erreichen der I/B/E/S-Analystenvorhersagen für die ordentlichen Betriebsergebnisse $I B \_B E_{-} M_{F, i, t}$ nachgewiesen werden. Zur Vereinfachung wird die Prävention von abschlusspolitischen Ergebnisspaltungen i. S. v. $\mathrm{H}_{1.3}$ nachfolgend nur beim Erreichen von nichtnegativen ordentlichen Betriebsergebnissen $B E_{-} N N_{F, i, t}$ untersucht.

\begin{tabular}{lccc}
\hline & Erw. Vz. & Bish. $U E W_{-} \Delta B E_{F, i, t+1}$ & Alt. $U E W_{-} \Delta B E_{F, i, t+1}$ \\
\hline$E A G B_{-} N_{i, t}$ & + & $-0,3146$ & 0,8056 \\
& & $(-2,11)$ & $(2,67)^{* * *}$ \\
$E A G B_{-} N_{i, t+1}$ & $-1,0248$ & 0,3451 \\
& $(-5,20)^{* * *}$ & $(1,14)$ \\
\hline Beob. & 325 & 325 \\
Adj. $\mathrm{R}^{2}$ & 0,0047 & 0,1862 \\
\hline
\end{tabular}

Restliche Terme aus Regressionsgleichung (17b) nicht abgedruckt.

t-Statistiken in Klammern. Variablendefinitionen in Tab. 19.

Test für Regressionskoeffizient von $E A G B \_N_{i, t}$ einseitig, ansonsten zweiseitig.

Tab. 43: Schätzung der hinreichenden Bedingung für die Existenz von abschlusspolitischen Ergebnisspaltungen beim Erhöhen der Ergebnisse aus den fortgeführten Geschäftsbereichen mit bisheriger und alternativer abhängiger Variable

Für die Prävention von abschlusspolitischen Ergebnisspaltungen beim Erhöhen der Ergebnisse aus des fortgeführten Geschäftsbereichen und von abschlusspolitischen Ergebnisspaltungen beim Erreichen von nichtnegativen ordentlichen Betriebsergebnissen sind in der Tab. 42 keine Testergebnisse für die bisherige Berechnung von $U E W \_\triangle B E_{F, i, t+1}$ angegeben, da die hinreichende Bedingung für $\mathrm{H}_{1.1}$ und $\mathrm{H}_{1.3}$ bislang nicht erfüllt war. Ohne die Existenz von abschlusspolitischen Ergebnisspaltungen kann deren Prävention schließlich nicht untersucht werden. Hinsichtlich der Prävention der nun nachgewiesenen Formen der abschlusspolitischen Ergebnisspaltungen werden zur Vollständigkeit auch die erste Möglichkeit bzw. Bedingung untersucht, obwohl diese von der alternativen Berechnung von $U E W_{-} \Delta B E_{F, i, t+1}$ nicht 


\begin{tabular}{llcc}
\hline & Koeff. & Bish. $U E W_{-} \Delta B E_{F, i, t+1}$ & Alt. $U E W_{-} \Delta B E_{F, i, t+1}$ \\
\hline$E A G B_{-} N_{i, t}$ & $\zeta_{15}$ & $-0,3683$ & 0,7075 \\
& & $(-2,66)^{* * *}$ & $(2,54)^{* *}$ \\
$B M_{-} G 1_{i, t}$ & $\zeta_{16}$ & $-0,0087$ & $-0,0133$ \\
& & $(-0,53)$ & $(-0,53)$ \\
$E A G B \_N_{i, t} \cdot B M_{-} G I_{i, t}$ & $\zeta_{17}$ & 1,1458 & 2,1023 \\
& & $(3,06)^{* * *}$ & $(3,69)^{* * *}$ \\
& $\zeta_{15}+\zeta_{17}$ & 0,7775 & 2,8098 \\
& & $(2,08)^{* *}$ & $(4,81)^{* * *}$ \\
$E A G B \_N_{i, t+1}$ & $\zeta_{18}$ & $-1,1110$ & 0,1768 \\
& & $(-5,70)^{* * *}$ & $(0,62)$ \\
\hline Beob. & & 325 & 325 \\
Adj. $\mathrm{R}^{2}$ & & 0,0017 & 0,1853 \\
\hline
\end{tabular}

Restliche Terme aus Regressionsgleichung (18b) nicht abgedruckt.

t-Statistiken in Klammern. Variablendefinitionen in Tab. 19.

Test für $\zeta_{15}+\zeta_{17}>0$ einseitig, ansonsten zweiseitige Tests.

Tab. 44: Schätzung der hinreichenden Bedingung für die Existenz von abschlusspolitischen Ergebnisspaltungen bei ineffizienten Unternehmensgrößen mit bisheriger und alternativer abhängiger Variable

berührt werden. ${ }^{1592}$ Hierbei zeigt sich, dass die untersuchten Präventivmaßnahmen zumeist versagen. ${ }^{1593}$ Konkret können abschlusspolitische Ergebnisspaltungen beim Erhöhen der Ergebnisse aus den fortgeführten Geschäftsbereichen durch keine der untersuchten Präventivmaßnahmen verhindert bzw. abgeschwächt werden. ${ }^{1594}$ Ebenso können abschlusspolitische Ergebnisspaltungen beim Erreichen von nichtnegativen ordentlichen Betriebsergebnissen durch keine der untersuchten Maßnahmen verhindert bzw. abgeschwächt werden und werden durch die Branchenspezialisierungen der Abschlussprüfer i. S. d. Alternativhypothese $\mathrm{H}_{2.8}$ sogar noch verstärkt. Allerdings können etwaige Wirkungen der Größen der Abschlussprüfer auf abschlusspolitische Ergebnisspaltungen beim Erreichen von nichtnegativen ordentlichen Betriebsergebnissen i. S. d. Alternativhypothese $\mathrm{H}_{2.7}$ nicht beurteilt werden, da die Regressionsgleichung für die notwendige Bedingung von $\mathrm{H}_{2.7}$ an einer perfekten Multikollinearität zwischen $E A G B \_N_{i, t}$ und $E A G B \_N_{i, t} \cdot B E_{-} N N_{F, i, t}$ leidet, die die Berechnung der t-Statistik von $E A G B \_N_{i, t} \cdot B E_{-} N N_{F, i, t}$ verhindert.

1592 Bei der ersten Möglichkeit bzw. Bedingung bildet $U E W_{-} B E_{F, i, t}$ die abhängige Variable.

1593 Die Schätzungen zur Prävention von abschlusspolitischen Ergebnisspaltungen mit der alternativen Berechnung von $U E W \_\Delta B E_{F, i, t+1}$ werden aus Platzgründen nicht wiedergegeben.

1594 Wie mit $\mathrm{H}_{2.7}$ und $\mathrm{H}_{2.8}$ postuliert und in der Fußzeile der Tab. 42 vermerkt, wirken sich die Größen der Abschlussprüfer respektive ihre Branchenspezialisierungen nicht auf abschlusspolitische Ergebnisspaltungen beim Erhöhen der Ergebnisse aus den fortgeführten Geschäftsbereichen aus. 


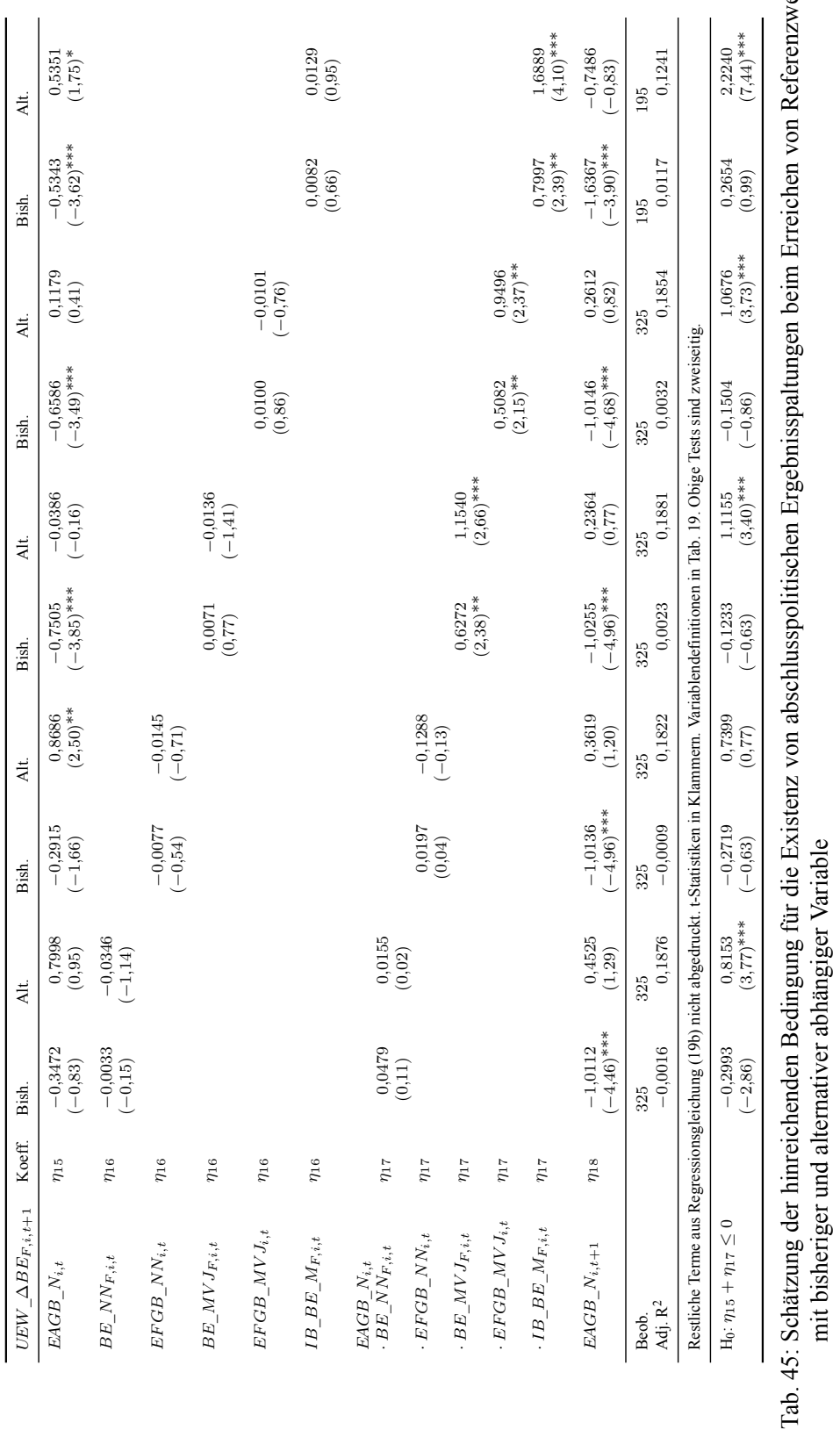


(4) In der vorliegenden Arbeit wurden abschlusspolitische Ergebnisspaltungen bei ineffizienten Unternehmensgrößen festgestellt. Dagegen konnten bei allen anderen untersuchten Motiven keine abschlusspolitischen Ergebnisspaltungen belegt werden. ${ }^{1595} \mathrm{Ei}$ ne andere Studie, die abschlusspolitische Ergebnisspaltungen bei ineffizienten Unternehmensgrößen untersucht, ist dem Verfasser der vorliegenden Arbeit nicht bekannt. Daher wird im Folgenden untersucht, ob die Feststellung von abschlusspolitischen Ergebnisspaltungen bei ineffizienten Unternehmensgrößen von der Definition der Interaktionsvariable abhängt, mit der ineffiziente Unternehmensgrößen gemessen werden. In der bisherigen Untersuchung wurden ineffiziente Unternehmensgrößen mit der Interaktionsvariable $B M_{-} G 1_{i, t}$ operationalisiert. $B M_{-} G 1_{i, t}$ wird der Wert 1 zugewiesen, falls der Buch- den Marktwert des Eigenkapitals übersteigt. Andernfalls wird $B M_{-} G 1_{i, t}$ mit dem Wert 0 kodiert. Die Idee hinter dieser Operationalisierung lautet, dass die Kapitalmärkte bei $B M \_G 1_{i, t}=1$ negative Gewinnerwartungen für die Unternehmen zu haben scheinen, die typischerweise aus ineffizienten Unternehmensgrößen resultieren. ${ }^{1596}$

Eine alternative und direktere - aber auch kompliziertere - Operationalisierung der ineffizienten Unternehmensgrößen könnte am Survivor Principle von Stigler (1958) anknüpfen. Das Survivor Principle besagt vereinfacht, dass innerhalb von wettbewerbsintensiven Branchen die Unternehmen mit effizienten Größen langfristig überleben. Das empirische Messverfahren des Survivor Principle basiert auf der Idee, dass die brancheninternen Marktanteile der Unternehmen mit den ineffizientesten Größen im Zeitverlauf am stärksten sinken, während die Marktanteile der Konkurrenten mit effizienten Größen steigen. ${ }^{1597}$ In Anlehnung an Stigler (1958) werden in der Tab. 46 die Unternehmensgrößen als prozentuale Anteile der Umsatzerlöse der fortgeführten Geschäftsbereiche, die Unternehmen in einem Geschäftsjahr erzielen, an den jährlichen Gesamtumsätzen der fortgeführten Geschäftsbereiche der CDAX-Technologieunternehmen aus der Stichprobe ermittelt. ${ }^{1598}$ In den Zeilen sind Größenintervalle angegeben, für die im oberen Bereich die Anteile der Umsatzerlöse

1595 Eine Ausnahme bildet der Robustheitstest (3) des vorliegenden Abschnitts. Bei diesem Robustheitstest wird die abhängige Variable $U E W \_\triangle B E_{F, i, t+1}$ alternativ berechnet, wodurch neben abschlusspolitischen Ergebnisspaltungen bei ineffizienten Unternehmensgrößem auch abschlusspolitische Ergebnisspaltungen zum Erhöhen der Ergebnisse aus den fortgeführten Geschäftsbereichen und abschlusspolitische Ergebnisspaltungen zum Erreichen von Referenzwerten belegt werden können.

1596 Der Zusammenhang zwischen negativen Gewinnerwartungen und ineffizienten Unternehmensgrößen wird im Abschnitt 9.2 ausführlich beschrieben.

1597 Siehe Stigler, G. J. (1958), S. 54-60 und den Unterabschnitt 5.2.5.

1598 Analog verwendet Stigler, G. J. (1958), S. 58-62 für Stahlhersteller die Anteile an der USamerikanischen Branchenkapazität und für Automobilhersteller die Anteile an den insgesamt in den USA hergestellten Automobilen. Da die Technologieunternehmen im CDAX sehr heterogene Produkte herstellen, werden in der vorliegenden Arbeit die Umsatzerlöse der fortgeführten Geschäftsbereiche als $\mathrm{Ma}$ für die Unternehmensgrößen verwendet. Um die Ausführungen kompakt zu halten, wird in der Tab. 46 und nachfolgend nur von den Umsatzerlösen gesprochen. 
kumuliert und im unteren Bereich die Unternehmen gezählt werden. ${ }^{1599}$ Beispielsweise bedeuten die Werte 0,8 und 11 in den beiden Bereichen oben links, dass diejenigen elf Unternehmen, die im Geschäftsjahr 2008 jeweils weniger als 0,10 Prozent an den gesamten Umsatzerlösen aller 60 Unternehmen erzielten, im selben Geschäftsjahr zusammen 0,8 Prozent der Umsatzerlöse erreichten.

\begin{tabular}{|c|c|c|c|c|c|c|c|}
\hline $\begin{array}{l}\text { Unternehmensgrößen in \% } \\
\text { der Branchenumsatzerlöse }\end{array}$ & 2008 & 2009 & 2010 & 2011 & 2012 & 2013 & $\Sigma$ \\
\hline & \multicolumn{7}{|c|}{ Kumulierte Anteile der Umsatzerlöse in \% } \\
\hline weniger als 0,10 & 0,8 & 0,5 & 0,5 & 0,5 & 0,5 & 0,3 & \\
\hline 0,10 bis 0,25 & 2,0 & 2,4 & 1,7 & 1,8 & 1,8 & 2,3 & \\
\hline 0,25 bis 0,50 & 3,9 & 3,8 & 5,3 & 4,1 & 4,4 & 3,4 & \\
\hline 0,50 bis 1,00 & 7,0 & 5,8 & 7,7 & 6,6 & 4,2 & 4,5 & \\
\hline 1,00 bis 2,00 & 10,0 & 9,6 & 8,3 & 10,6 & 9,3 & 9,0 & \\
\hline mehr als 2,00 & 76,3 & 77,9 & 76,5 & 76,4 & 79,8 & 80,5 & \\
\hline \multirow[t]{2}{*}{$\Sigma$} & 100,0 & 100,0 & 100,0 & 100,0 & 100,0 & 100,0 & \\
\hline & \multicolumn{7}{|c|}{ Anzahl Unternehmen } \\
\hline weniger als 0,10 & 11 & 8 & 9 & 9 & 9 & 5 & 51 \\
\hline 0,10 bis 0,25 & 12 & 14 & 11 & 11 & 11 & 14 & 73 \\
\hline 0,25 bis 0,50 & 10 & 10 & 14 & 11 & 12 & 9 & 66 \\
\hline 0,50 bis 1,00 & 11 & 9 & 10 & 10 & 6 & 7 & 53 \\
\hline 1,00 bis 2,00 & 8 & 7 & 5 & 7 & 6 & 6 & 39 \\
\hline mehr als 2,00 & 8 & 9 & 8 & 6 & 6 & 6 & 43 \\
\hline$\Sigma$ & 60 & 57 & 57 & 54 & 50 & 47 & 325 \\
\hline
\end{tabular}

Tab. 46: Verteilung der Umsatzanteile und der Anzahl von Technologieunternehmen im CDAX nach Unternehmensgrößen (in Anlehnung an: Stigler, G. J. (1958), S. 58.)

Fraglich ist, wie anhand der Tab. 46 ineffizienten Unternehmensgrößen ermittelt werden können, für die mit der Alternativhypothese $\mathrm{H}_{1.2}$ auf der S. 115 abschlusspolitische Ergebnisspaltungen assoziiert werden. Gemäß dem Survivor Principle sind diejenigen Unternehmensgrößen ineffizient, die im Zeitverlauf Marktanteile verlieren. Bei einer gemeinsamen Betrachtung des oberen und unteren Bereichs der Tab. 46 zeigt sich, dass im Zeitverlauf vor allem Unternehmen, die weniger als 0,10 Prozent der Branchenumsatzerlöse erzielen, sowie Unternehmen, die zwischen 0,50 und 1,00 Prozent der Branchenumsatzerlöse erreichen, Marktanteile verlieren oder diese Größenklassen verlassen. Daher werden diesen $51+53=104$ Beobachtungen ineffiziente Unternehmensgrößen unterstellt und mit einer neuen Interaktionsvariable $G R_{-} I E F F_{F, i, t}$ getes-

1599 In Anlehnung an Weiss, L. W. (1964), S. 247 f. werden die Größenintervalle so gewählt, dass zu Beginn des Beobachtungszeitraums in die Größenintervalle etwa gleich viele Unternehmen fallen und die Obergrenzen der Intervalle typischerweise doppelt so hoch wie die Untergrenzen sind. 
tet, ${ }^{1600}$ ob bei ihnen abschlusspolitische Ergebnisspaltungen wahrscheinlicher als bei den restlichen Beobachtungen sind. Hierfür wird der nominalskalierten Interaktionsvariable $G R \_I E F F_{F, i, t}$ der Wert 1 zugewiesen, falls eine Beobachtung weniger als 0,10 Prozent oder zwischen 0,50 und 1,00 Prozent der jährlichen Branchenumsatzerlöse erzielt. Andernfalls wird $G R \_I E F F_{F, i, t}$ mit dem Wert 0 kodiert.

Die Tab. 47 und die Tab. 48 zeigen die Schätzungen der notwendigen respektive der hinreichenden Bedingung für abschlusspolitische Ergebnisspaltungen mit der bisherigen Interaktionsvariable für ineffiziente Unternehmensgrößen $B M_{-} G 1_{i, t}$ und der alternativen Interaktionsvariable $G R \_I E F F_{F, i, t}$. Inhaltlich bleiben die Testergebnisse durch die an das Survivor Principle angelehnte Interaktionsvariable unverändert, da auch mit $G R_{-} I E F F_{F, i, t}$ die notwendige und die hinreichende Bedingung für abschlusspolitische Ergebnisspaltungen bei ineffizienten Unternehmensgrößen erfüllt sind. ${ }^{1601}$

\begin{tabular}{|c|c|c|c|}
\hline \multirow[b]{2}{*}{$U E W_{-} B E_{F, i, t}$} & \multirow[b]{2}{*}{ Koeff. } & \multicolumn{2}{|c|}{ Interaktionsvariable } \\
\hline & & $B M_{-} G 1_{i, t}$ & $G R \_I E F F_{F, i, t}$ \\
\hline$E A G B_{-} N_{i, t}$ & $\zeta_{1}$ & $\begin{array}{c}-1,1723 \\
(-6,57)^{* * *}\end{array}$ & $\begin{array}{c}-1,2418 \\
(-5,65)^{* * *}\end{array}$ \\
\hline$B M_{-} G 1_{i, t}$ & $\zeta_{2}$ & $\begin{array}{l}-0,0088 \\
(-0,74)\end{array}$ & \\
\hline$G R \_I E F F_{F, i, t}$ & $\zeta_{2}$ & & $\begin{array}{l}0,0006 \\
(0,09)\end{array}$ \\
\hline$E A G B \_N_{i, t} \cdot B M_{-} G 1_{i, t}$ & $\zeta_{3}$ & $\begin{array}{l}-2,6234 \\
(-1,38)\end{array}$ & \\
\hline$E A G B \_N_{i, t} \cdot G R \_I E F F_{F, i, t}$ & $\zeta_{3}$ & & $\begin{array}{c}-2,4369 \\
(-3,71)^{* * *}\end{array}$ \\
\hline Beob. & & 325 & 325 \\
\hline Adj. $R^{2}$ & & 0,2832 & 0,2785 \\
\hline \multicolumn{4}{|c|}{$\begin{array}{l}\text { Restliche Terme aus Regressionsgleichung (18a) nicht abgedruckt. } \\
\text { t-Statistiken in Klammern. Variablendefinitionen in Tab. } 19 . \\
\text { Obige Tests sind zweiseitig. }\end{array}$} \\
\hline $\mathrm{H}_{0}: \zeta_{1}+\zeta_{3} \geq 0$ & & $\begin{array}{c}-3,7956 \\
(-1,95)^{* *}\end{array}$ & $\begin{array}{c}-3,6787 \\
(-6,10)^{* * *}\end{array}$ \\
\hline
\end{tabular}

Tab. 47: Schätzung der notwendigen Bedingung für die Existenz von abschlusspolitischen Ergebnisspaltungen mit bisherigen und alternativen Interaktionsvariablen für ineffiziente Unternehmensgrößen

1600 Der Variablenname $G R_{-} I E F F_{F, i, t}$ kürzt den Ausdruck ,ineffiziente Größen“ ab.

1601 Im Übrigen bleiben die notwendige und die hinreichende Bedingung auch dann erfüllt, wenn nur Unternehmen, die weniger als 0,10 Prozent der Branchenumsatzerlöse erzielen, oder nur Unternehmen, die zwischen 0,50 und 1,00 Prozent der Branchenumsatzerlöse erreichen, als Unternehmen mit ineffizienten Größen definiert werden. Auf die detaillierte Wiedergabe dieser Testergebnisse wird verzichtet. 


\begin{tabular}{|c|c|c|c|}
\hline \multirow[b]{2}{*}{$U E W \_\Delta B E_{F, i, t+1}$} & \multirow[b]{2}{*}{ Koeff. } & \multicolumn{2}{|c|}{ Interaktionsvariable } \\
\hline & & $B M_{-} G 1_{i, t}$ & $G R \_I E F F_{F, i, t}$ \\
\hline$E A G B \_N_{i, t}$ & $\zeta_{15}$ & $\begin{array}{c}-0,3683 \\
(-2,66)^{* * *}\end{array}$ & $\begin{array}{c}-0,3863 \\
(-3,00)^{* * *}\end{array}$ \\
\hline$B M_{-} G 1_{i, t}$ & $\zeta_{16}$ & $\begin{array}{l}-0,0087 \\
(-0,53)\end{array}$ & \\
\hline$G R \_I E F F_{F, i, t}$ & $\zeta_{16}$ & & $\begin{array}{l}0,0052 \\
(0,58)\end{array}$ \\
\hline$E A G B \_N_{i, t} \cdot B M_{-} G 1_{i, t}$ & $\zeta_{17}$ & $\begin{array}{c}1,1458 \\
(3,06)^{* * *}\end{array}$ & \\
\hline$E A G B \_N_{i, t} \cdot G R \_I E F F_{F, i, t}$ & $\zeta_{17}$ & & $\begin{array}{c}1,9655 \\
(2,23)^{* *}\end{array}$ \\
\hline$E A G B_{-} N_{i, t+1}$ & $\zeta_{18}$ & $\begin{array}{c}-1,1110 \\
(-5,70)^{* * *} \\
\end{array}$ & $\begin{array}{c}-1,0195 \\
(-5,12)^{* * *} \\
\end{array}$ \\
\hline Beob. & & 325 & 325 \\
\hline Adj. $R^{2}$ & & 0,0017 & 0,0037 \\
\hline \multicolumn{4}{|c|}{$\begin{array}{l}\text { Restliche Terme aus Regressionsgleichung (18b) nicht abgedruckt. } \\
\text { t-Statistiken in Klammern. Variablendefinitionen in Tab. } 19 . \\
\text { Obige Tests sind zweiseitig. }\end{array}$} \\
\hline $\mathrm{H}_{0}: \zeta_{15}+\zeta_{17} \leq 0$ & & $\begin{array}{c}0,7775 \\
(2,08)^{* *}\end{array}$ & $\begin{array}{c}1,5792 \\
(1,79)^{* *}\end{array}$ \\
\hline
\end{tabular}

Tab. 48: Schätzung der hinreichenden Bedingung für die Existenz von abschlusspolitischen Ergebnisspaltungen mit bisherigen und alternativen Interaktionsvariablen für ineffiziente Unternehmensgrößen

Insgesamt bestätigen die vier Robustheitstests die in der Tab. 35 und der Tab. 41 zusammengefassten Testergebnisse. Die einzige Ausnahme bildet der Robustheitstest (3), bei dem die abhängige Variable mit einer alternativen Methode berechnet wurde. Mit dieser alternativen Methode können neben abschlusspolitischen Ergebnisspaltungen bei ineffizienten Unternehmensgrößen auch abschlusspolitische Ergebnisspaltungen beim Erhöhen der Ergebnisse aus den fortgeführten Geschäftsbereichen und abschlusspolitische Ergebnisspaltungen zum Erreichen von Referenzwerten festgestellt werden, die durch Präventivmaßnahmen kaum zu verhindern sind.

\subsection{Ergänzende Analysen}

Mit den folgenden ergänzenden Analysen werden naheliegende Erweiterungen der bisherigen empirischen Erkenntnisse untersucht. Im Unterschied zum Abschnitt 11.1 soll mit 
diesen Analysen nicht die Robustheit der bisherigen empirischen Erkenntnisse eruiert, sondern die vorhanden Daten explorativ untersucht werden: ${ }^{162}$

(1) In der vorliegenden Arbeit wurden für Vorstandsvergütungen i. S. v. $\mathrm{H}_{2.2}$, Kapitalstrukturen i. S. v. $\mathrm{H}_{2.4}$ und Nichtprüfungshonorare i. S. v. $\mathrm{H}_{2.6}$ schwächere abschlusspolitische Ergebnisspaltungen postuliert. ${ }^{1603}$ Die Prävention von abschlusspolitischen Ergebnisspaltungen wurde für $\mathrm{H}_{2.2}, \mathrm{H}_{2.4}$ und $\mathrm{H}_{2.6}$ bewusst nicht postuliert, da diese Alternativhypothesen mit verhältnisskalierten Interaktionsvariablen operationalisiert werden, für die es keine theoretisch fundierten Werte gibt, ab denen vollständige Präventionen zu erwarten sind. ${ }^{1604}$ Behelfsweise wurde untersucht, ob abschlusspolitische Ergebnisspaltungen bei einer Standardabweichung über dem Mittelwert der verhältnisskalierten Interaktionsvariablen für $\mathrm{H}_{2.2}, \mathrm{H}_{2.4}$ und $\mathrm{H}_{2.6}$ statistisch signifikant schwächer als bei einer Standardabweichung unter dem Mittelwert sind. ${ }^{1605}$ Diese Untersuchung entspricht dem Test (3) in der Fußzeile der Tab. 39. Da die Nullhypothesen des Tests (3) mit den verhältnisskalierten Interaktionsvariablen $V V E R G_{i, t}$, $V S G_{F, i, t}$ und $P H_{-} G H_{i, t}$ abgelehnt werden können, scheinen Vorstandsvergütungen i. S. v. $\mathrm{H}_{2.2}$, Kapitalstrukturen i. S. v. $\mathrm{H}_{2.4}$ und Nichtprüfungshonorare i. S. v. $\mathrm{H}_{2.6}$ abschlusspolitische Ergebnisspaltungen bei ineffizienten Unternehmensgrößen abzuschwächen. Diese Abschwächungen sind aber nicht mit einer Prävention von abschlusspolitischen Ergebnisspaltungen gleichbedeutend. Vielmehr können interne Corporate-Governance-Mechanismen abschlusspolitische Ergebnisspaltungen statistisch signifikant abschwächen, aber die abschlusspolitischen Ergebnisspaltungen trotzdem statistisch signifikant bleiben. Ein derartiger Fortbestand von signifikanten abschlusspolitischen Ergebnisspaltungen trotz einer signifikanten Abschwächung durch Corporate-Governance-Mechanismen liegt in der Tab. 39 für die Interaktionsvariablen $V V E R G_{i, t}$ und $P H_{-} G H_{i, t}$ vor. In den dortigen Tests (2) kann selbst bei hohen Werten $V V E R G_{i, t, H}$ und $P H_{-} G H_{i, t, H}$ die Nullhypothese von fortbestehenden abschlusspolitischen Ergebnisspaltungen bei ineffizienten Unternehmensgrößen nicht abgelehnt werden. Somit werden die abschlusspolitischen Ergebnisspaltungen bei ineffizienten Unternehmensgrößen mit hohen Werten der verhältnisskalierten Interaktionsvariablen $V V E R G_{i, t}$ und $P H_{-} G H_{i, t}$ abgeschwächt, aber nicht vollständig

1602 Bortz, J./Döring, N. (2006), S. 352-391 diskutieren die explorative Hypothesengewinnung und Theoriebildung ausführlich.

1603 Die Alternativhypothesen $\mathrm{H}_{2.2}, \mathrm{H}_{2.4}$ und $\mathrm{H}_{2.6}$ sind in der Tab. 36 zusammengetragen.

1604 Dagegen werden für Aufsichtsräte i. S. v. $\mathrm{H}_{2.1}$, Eigentümerstrukturen i. S. v. $\mathrm{H}_{2.3}$ und Prüfungshonorare i. S. v. $\mathrm{H}_{2.5}$ eine vollständige Prävention von abschlusspolitischen Ergebnisspaltungen postuliert, da diese Alternativhypothesen mit nominalskalierten Interaktionsvariablen operationalisiert werden, für die aufgrund theoretischer Überlegungen beim Wert 1 eine vollständige Prävention erwartet wird. Beispielsweise ist unklar, ab welcher Höhe der Verschuldungsgrade $V S G_{F, i, t}$ i. S. v. $\mathrm{H}_{2.4}$ abschlusspolitische Ergebnisspaltungen vollständig verhindert werden können, wohingegen hierzu einzelne Großaktionäre $G R A K_{i, t}=1$ i. S. v. $\mathrm{H}_{2.3}$ ausreichen können. Die Alternativhypothesen $\mathrm{H}_{2.1}, \mathrm{H}_{2.3}$ und $\mathrm{H}_{2.5}$ sind in der Tab. 36 zusammengetragen.

1605 Siehe ausführlich den Abschnitt 10.1. 
verhindert. Ebenso schwächt ein hoher Wert für die Branchenspezialisierungen der Abschlussprüfer ${ }^{1606}$ APSPEZ $Z_{i, t, H}$ im Test (2) der Tab. 39 abschlusspolitische Ergebnisspaltungen bei ineffizienten Unternehmensgrößen statistisch signifikant ab, verhindert diese aber ebenfalls nicht.

Ergänzend wird nun untersucht, ob die zweite Möglichkeit zur Abschwächung von abschlusspolitischen Ergebnisspaltungen bei ineffizienten Unternehmensgrößen mit hohen Werten der verhältnisskalierten Interaktionsvariablen $V V E R G_{i, t}$ und $P H \_G H_{i, t}$ in einer entsprechenden vollständigen Prävention mündet. In der Tab. 49 kann die Nullhypothese von fortbestehenden abschlusspolitischen Ergebnisspaltungen bei ineffizienten Unternehmensgröße im Test (3) mit keiner der Interaktionsvariablen $V V E R G_{i, t}$ und $\mathrm{PH}_{-} \mathrm{GH}_{i, t}$ abgelehnt werden. Folglich führt auch die zweite Möglichkeit zur Abschwächung von abschlusspolitischen Ergebnisspaltungen bei ineffizienten Unternehmensgrößen mit hohen Werten $V V E R G_{i, t, H}$ und $P H_{-} G H_{i, t, H}$ nicht zu einer entsprechenden vollständigen Prävention. Ebenso verhindert ein hoher Wert APSPEZ $Z_{i, t, H}$ im Test (3) der Tab. 49 abschlusspolitische Ergebnisspaltungen bei ineffizienten Unternehmensgrößen nicht vollständig. Zusammengefasst schwächen Vorstandsvergütungen i. S. v. $\mathrm{H}_{2.2}$, Nichtprüfungshonorare i. S. v. $\mathrm{H}_{2.6}$ und Branchenspezialisierungen der Abschlussprüfer i. S. v. $\mathrm{H}_{2.8}$ abschlusspolitische Ergebnisspaltungen bei ineffizienten Unternehmensgrößen zwar ab, verhindern sie aber nicht vollständig.

\begin{tabular}{|c|c|c|c|c|}
\hline \multicolumn{2}{|l|}{ Regressionsgleichung (25b) } & \multicolumn{3}{|c|}{ Interaktionsvariable } \\
\hline$U E W \_\Delta B E_{F, i, t+1}$ & Koeff. & $\overline{V V E R G_{i, t}}$ & $P H_{-} G H_{i, t}$ & $A P S P E Z_{i, t}$ \\
\hline$E A G B_{-} N_{i, t}$ & $\lambda_{19}$ & $\begin{array}{l}-1,2227 \\
(-0,83)\end{array}$ & $\begin{array}{c}-1,0584 \\
(-2,09)^{* *}\end{array}$ & $\begin{array}{l}-0,1773 \\
(-0,80)\end{array}$ \\
\hline$B M_{-} G 1_{i, t}$ & $\lambda_{20}$ & $\begin{array}{l}0,0349 \\
(0,75)\end{array}$ & $\begin{array}{c}0,0546 \\
(1,29)\end{array}$ & $\begin{array}{l}-0,0202 \\
(-0,89)\end{array}$ \\
\hline$V V E R G_{i, t}$ & $\lambda_{21}$ & $\begin{array}{c}0,0006 \\
(1,96)^{*}\end{array}$ & & \\
\hline$P H \_G H_{i, t}$ & $\lambda_{21}$ & & $\begin{array}{c}0,0005 \\
(2,31)^{* *}\end{array}$ & \\
\hline$A P S P E Z_{i, t}$ & $\lambda_{21}$ & & & $\begin{array}{l}0,0003 \\
(0,89)\end{array}$ \\
\hline$E A G B \_N_{i, t} \cdot B M_{-} G 1_{i, t}$ & $\lambda_{22}$ & $\begin{array}{l}7,1347 \\
(1,18)\end{array}$ & $\begin{array}{l}68,2742 \\
(1,17)\end{array}$ & $\begin{array}{l}2,8479 \\
(0,74)\end{array}$ \\
\hline $\begin{array}{l}E A G B \_N_{i, t} \\
\cdot V V E R G_{i, t}\end{array}$ & $\lambda_{23}$ & $\begin{array}{l}0,0112 \\
(0,60)\end{array}$ & & \\
\hline$\cdot P H_{-} G H_{i, t}$ & $\lambda_{23}$ & & $\begin{array}{c}0,0090 \\
(1,67)^{*}\end{array}$ & \\
\hline
\end{tabular}

Fortsetzung auf der nächsten Seite

1606 Die zugehörige Alternativhypothese $\mathrm{H}_{2.8}$ ist in der Tab. 36 wiedergegeben. 


\begin{tabular}{|c|c|c|c|c|}
\hline \multicolumn{2}{|l|}{ Regressionsgleichung (25b) } & \multicolumn{3}{|c|}{ Interaktionsvariable } \\
\hline$U E W \_\Delta B E_{F, i, t+1}$ & Koeff. & $V V E R G_{i, t}$ & $P H_{-} G H_{i, t}$ & $A P S P E Z_{i, t}$ \\
\hline - $A P S P E Z_{i, t}$ & $\lambda_{23}$ & & & $\begin{array}{l}-0,0101 \\
(-1,19)\end{array}$ \\
\hline $\begin{array}{l}B M_{-} G 1_{i, t} \\
\cdot V V E R G_{i, t}\end{array}$ & $\lambda_{24}$ & $\begin{array}{l}-0,0006 \\
(-0,92)\end{array}$ & & \\
\hline$\cdot P H_{-} G H_{i, t}$ & $\lambda_{24}$ & & $\begin{array}{c}-0,0008 \\
(-1,69)^{*}\end{array}$ & \\
\hline$\cdot A P S P E Z_{i, t}$ & $\lambda_{24}$ & & & $\begin{array}{c}0,0007 \\
(0,99)\end{array}$ \\
\hline $\begin{array}{l}E A G B \_N_{i, t} \cdot B M_{-} G 1_{i, t} \\
\cdot V V E R G_{i, t}\end{array}$ & $\lambda_{25}$ & $\begin{array}{l}-0,0719 \\
(-1,03)\end{array}$ & & \\
\hline$\cdot P H_{-} G H_{i, t}$ & $\lambda_{25}$ & & $\begin{array}{l}-0,6840 \\
(-1,16)\end{array}$ & \\
\hline - $A P S P E Z_{i, t}$ & $\lambda_{25}$ & & & $\begin{array}{l}-0,0319 \\
(-0,32)\end{array}$ \\
\hline$E A G B_{-} N_{i, t+1}$ & $\lambda_{26}$ & $\begin{array}{c}-1,3685 \\
(-2,75)^{* * *}\end{array}$ & $\begin{array}{c}-1,4748 \\
(-3,34)^{* * *}\end{array}$ & $\begin{array}{c}-1,2852 \\
(-2,56)^{* *}\end{array}$ \\
\hline Beob. & & 325 & 325 & 325 \\
\hline Adj. $R^{2}$ & & 0,0037 & 0,0052 & 0,0038 \\
\hline
\end{tabular}

Restliche Terme aus Regressionsgleichung (25b) nicht abgedruckt.

t-Statistiken in Klammern. Variablendefinitionen in Tab. 19. Obige Tests sind zweiseitig.

(1) $\mathrm{H}_{0}: \lambda_{19}+\lambda_{22}+\left(\lambda_{23}+\lambda_{25}\right)\left\{P R V \_2\right\}_{N} \geq 0$

$\begin{array}{lll}2,7216 & 28,4102 & 2,6644 \\ (1,07) & (1,16) & (0,70)\end{array}$

(2) $\mathrm{H}_{0}: \lambda_{19}+\lambda_{22}+\left(\lambda_{23}+\lambda_{25}\right)\left\{P R V \_2\right\}_{H} \geq 0$

$\begin{array}{lcc}0,4316 & 2,0292 & 1,2260 \\ (1,59) & (1,30) & (2,13)\end{array}$

(3) $\mathrm{H}_{0}:\left(\lambda_{23}+\lambda_{25}\right)\left(\left\{P R V_{-}\right\}_{H}-\left\{P R V_{-} 2\right\}_{N}\right) \geq 0$

$$
\begin{array}{lll}
-2,2900 & -26,3809 & -1,4385 \\
(-0,84) & (-1,15) & (-0,43)
\end{array}
$$

(4) $\mathrm{H}_{0}:\left(\lambda_{23}+\lambda_{25}\right)\left(A P S P E Z_{i, t, H}-A P S P E Z_{i, t, N}\right)=0 \quad-1,4385$

$(-0,43)$

Tab. 49: Schätzung der zweiten Möglichkeit zur Prävention von abschlusspolitischen Ergebnisspaltungen bei ineffizienten Unternehmensgrößen mit verhältnisskalierten Interaktionsvariablen

(2) Neben abschlusspolitischen Ergebnisspaltungen wird in der Fachliteratur auffällig oft ein abschlusspolitischer Ausweis von aufgegebenen Geschäftsbereichen befürch- 
tet. ${ }^{1607}$ Während bei abschlusspolitischen Ergebnisspaltungen mit aufgegebenen Geschäftsbereichen die Zuordnungen von Aufwendungen bzw. Erträgen zu den fortgeführten oder aufgegebenen Geschäftsbereichen ermessensbehaftet sind, beziehen sich die Ermessensspielräume beim abschlusspolitischen Ausweis von aufgegebenen Geschäftsbereichen auf Auslegungen der Definition eines aufgegebenen Geschäftsbereichs. ${ }^{1608}$ Diese Definition eines aufgegebenen Geschäftsbereichs ist in IFRS 5.32 normiert und greift bspw. auf das Kriterium zurück, ob ein Unternehmensteil einen gesonderten, wesentlichen Geschäftszweig darstellt. ${ }^{1609}$ Dieses Kriterium können Vorstände entsprechend ihrer abschlusspolitischen Zielsetzungen auslegen, ${ }^{1610}$ weshalb Kümpel/Straatmann (2005) anmerken: „Insgesamt sind also die Klassifikationskriterien als unscharf zu beurteilen, so dass IFRS 5 ein Spielfeld für eine entsprechende Bilanzpolitik darstellt. Dies kann insbesondere für die aufzugebenden Geschäftsbereiche genutzt werden, indem z. B. Geschäftsbereiche mit einer schlechten Ertragslage als held for sale eingestuft werden und somit eine Ausgliederung von Ergebnis und Cash-flow aus den fortgeführten Aktivitäten erfolgt. Ob eine derartige Bilanzpolitik betrieben wird, bleibt abzuwarten. “1611 Ferner zeigen Skousen/Sun/Wu (2019) für den US-amerikanischen Rechtsraum, dass weniger fähige Vorstände Geschäftsbereiche abschlusspolitisch als aufgegebene Geschäftsbereiche deklarieren. Dieser abschlusspolitische Ausweis von aufgegebenen Geschäftsbereichen ist unter der APB Opinion No. 30, die dem IFRS 5 ähnelt, sogar besonders stark ausgeprägt. ${ }^{1612}$ Um zu testen, ob aufgegebene Geschäftsbereiche abschlusspolitisch ausgewiesen werden, werden anstatt den verhältnisskalierten Testvariablen $E A G B_{i, t}, E A G B_{-} N_{i, t}$ und $E A G B_{-} P_{i, t}$ die folgenden nominalskalierten Testvariablen verwendet:

1607 Siehe Albrecht, M. (2016), S. 165; Anders, G. (2016), S. 270; Blom, M./Baur, D. (2006), S. 897; Böcking, H.-J./Kiefer, M. (2016), IFRS 5, Tz. 65; Böcking, H.-J./Worret, D. (2016), S. 123; Bödecker, A./Fischer, F./Teuteberg, T. (2016), S. 972; Curtis, A./McVay, S./Wolfe, M. (2014), S. 194; Gusinde, P. (2000), S. 239-241; Hoffmann, W.-D./Lüdenbach, N. (2004), S. 2008; Keitz, I. v./Heyd, R. (2017), IFRS 5, Tz. 3; Kümpel, T./Straatmann, L. (2005), S. 142/146; Küting, K./Wirth, J. (2006), S. 728; Lüdenbach, N./Hoffmann, W.-D./Freiberg, J. (2020), § 29, Tz. 29; Poerschke, K. (2006), S. 56/168-170; Respondek, R. (2009), S. 84-86; Rogler, S./Tettenborn, M./Straub, S. V. (2012), S. 384; Schildbach, T. (2005), S. 559; Schlaak, W. (2014), S. 231 f.; Scholvin, P./Ramscheid, M. (2016), § 28, Tz. 101; Voelkner, B. (2005), S. 79; Zülch, H./Nellessen, T. (2008), S. 407. Analog Eisenman, S./Akresh, M. S./Snow, C. (1979), S. 27 f.; Morris, J. M. (1984), S. 173; Smith, T. (1996), S. 54.

1608 Vgl. Respondek, R. (2009), S. 66/84 f. Ungefähr Coutinho e Silva, A. H. et al. (2018), S. 30 f.

1609 So IFRS 5.32 Buchst. (a). Siehe weiterführend den Unterabschnitt 4.1.1.

1610 Hinsichtlich der Tab. 9 können diese Auslegungen als faktische Wahlrechte interpretiert werden.

1611 Kümpel, T./Straatmann, L. (2005), S. 146. Ähnlich Albrecht, M. (2016), S. 165; Böcking, H.-J./Kiefer, M. (2016), IFRS 5, Tz. 65; Gusinde, P. (2000), S. 239-241; Keitz, I. v./Heyd, R. (2017), IFRS 5, Tz. 3; Morris, J. M. (1984), S. 173; Poerschke, K. (2006), S. 56/168; Respondek, R. (2009), S. 66/84-86; Rogler, S./Tettenborn, M./Straub, S. V. (2012), S. 384. Andere Ansicht bei Küting, K./Wirth, J. (2006), S. 725, Fn. 62, die sich explizit von Kümpel, T./Straatmann, L. (2005), S. 146 abgrenzen.

1612 Zum Vorhergehenden Skousen, C./Sun, L./Wu, K. (2019), S. 124-128. 
- $E A G B_{-} E_{i, t}$ wird der Wert 1 zugewiesen, falls in einem Geschäftsjahr mindestens ein aufgegebener Geschäftsbereich ausgewiesen wird. ${ }^{1613}$ Andernfalls erhält $E A G B \_E_{i, t}$ den Wert 0 .

- $E A G B \_N \_E_{i, t}$ wird der Wert 1 zugewiesen, falls das Ergebnis aus den aufgegebenen Geschäftsbereichen negativ ist. Andernfalls erhält $E A G B_{-} N_{-} E_{i, t}$ den Wert 0 .

- $E A G B_{-} P_{-} E_{i, t}$ wird der Wert 1 zugewiesen, falls das Ergebnis aus den aufgegebenen Geschäftsbereichen positiv ist. Andernfalls erhält $E A G B_{-} P_{-} E_{i, t}$ den Wert 0.

Allerdings sind bereits die notwendigen Bedingungen für den abschlusspolitischen Ausweis von aufgegebenen Geschäftsbereichen beim Erhöhen der Ergebnisse aus den fortgeführten Geschäftsbereichen, beim Erreichen von Referenzwerten, bei Cookie Jars oder bei Big Baths nicht erfüllt. Entgegen den Bedenken in der Fachliteratur wird in der vorliegenden Arbeit somit kein abschlusspolitischer Ausweis von aufgegebenen Geschäftsbereichen festgestellt. ${ }^{1614}$ Exemplarisch ist in der Tab. 50 die notwendige Bedingung für einen abschlusspolitischen Ausweis von aufgegebenen Geschäftsbereichen zum Erhöhen der Ergebnisse aus den fortgeführten Geschäftsbereichen und in der Tab. 51 die notwendige Bedingung für abschlusspolitische Ergebnisspaltungen zum Verschleiern von ineffizienten Unternehmensgrößen wiedergegeben.

\begin{tabular}{lcccc}
\hline$U E W_{-} B E_{F, i, t}$ & Erw. Vz. & $(15)$ & $(16)$ & $(17 \mathrm{a})$ \\
\hline$E A G B_{-} E_{i, t}$ & - & 0,0328 & & \\
& & $(1,87)$ & & \\
$E A G B \_N_{-} E_{i, t}$ & - & & 0,0454 & 0,0437 \\
& & & $(1,64)$ & $(1,60)$ \\
$E A G B_{-} P_{-} E_{i, t}$ & - & & 0,0176 & \\
& & & $(1,17)$ & \\
\hline Beob. & & 325 & 325 & 325 \\
Adj. $\mathrm{R}^{2}$ & & 0,2434 & 0,2449 & 0,2448 \\
\hline
\end{tabular}

Restliche Terme aus Regressionsgleichungen (15), (16) und 17a) nicht abgedruckt. t-Statistiken in Klammern. Variablendefinitionen in Tab. 19. Obige Tests sind einseitig.

Tab. 50: Schätzung der notwendigen Bedingung für die Existenz von abschlusspolitisch ausgewiesenen aufgegebenen Geschäftsbereichen zum Erhöhen der Ergebnisse aus den fortgeführten Geschäftsbereichen

1613 Ebenso Skousen, C./Sun, L./Wu, K. (2019), S. 118/131. Die Endung „_E“ des Variablennamens $E A G B \_E_{i, t}$ bedeutet, dass ein aufgegebener Geschäftsbereich existiert.

1614 Gleiches Ergebnis bei Coutinho e Silva, A. H. et al. (2018), S. 30 f; Curtis, A./McVay, S./Wolfe, M. (2014), S. 191/198. 


\begin{tabular}{|c|c|c|c|}
\hline$U E W_{-} B E_{F, i, t}$ & Koeff. & (18a) & (18a) \\
\hline$E A G B_{-} E_{i, t}$ & $\zeta_{1}$ & $\begin{array}{l}0,0227 \\
(1,55)\end{array}$ & \\
\hline$E A G B \_N \_E_{i, t}$ & $\zeta_{1}$ & & $\begin{array}{c}0,0413 \\
(1,99)^{*}\end{array}$ \\
\hline$B M_{-} G 1_{i, t}$ & $\zeta_{2}$ & $\begin{array}{l}-0,0094 \\
(-0,82)\end{array}$ & $\begin{array}{l}-0,0087 \\
(-0,73)\end{array}$ \\
\hline$E A G B \_E_{i, t} \cdot B M_{-} G 1_{i, t}$ & $\zeta_{3}$ & $\begin{array}{c}0,0440 \\
(1,18)\end{array}$ & \\
\hline$E A G B \_N \_E_{i, t} \cdot B M_{-} G I_{i, t}$ & $\zeta_{3}$ & & $\begin{array}{c}0,0112 \\
(0,22)\end{array}$ \\
\hline Beob. & & 325 & 325 \\
\hline Adj. $R^{2}$ & & 0,2446 & 0,2409 \\
\hline
\end{tabular}

Restliche Terme aus Regressionsgleichungen (18a) nicht abgedruckt. t-Statistiken in Klammern. Variablendefinitionen in Tab. 19.

Obige Tests sind zweiseitig.

\begin{tabular}{ccc}
\hline $\mathrm{H}_{0}: \zeta_{1}+\zeta_{3} \geq 0$ & 0,0667 & 0,0525 \\
& $(1,57)$ & $(0,85)$ \\
\hline
\end{tabular}

Tab. 51: Schätzung der notwendigen Bedingung für die Existenz von abschlusspolitisch ausgewiesenen aufgegebenen Geschäftsbereichen bei ineffizienten Unternehmensgrößen

(3) In der vorliegenden Arbeit wurde postuliert, dass sich abschlusspolitische Ergebnisspaltungen durch geeignete interne Corporate-Governance-Mechanismen und Qualitätsmerkmale der Abschlussprüfungen verhindern lassen. Umgekehrt ist es denkbar, dass fehlende oder ungeeignete Corporate-Governance-Mechanismen und Qualitätsmerkmale der Abschlussprüfungen abschlusspolitische Ergebnisspaltungen begünstigen oder für Vorstände sogar diesbezügliche Anreize darstellen. ${ }^{1615}$ Daher wird im Folgenden untersucht, ob abschlusspolitische Ergebnisspaltungen bei fehlenden oder ungeeigneten Corporate-Governance-Mechanismen und Qualitätsmerkmalen der Abschlussprüfungen wahrscheinlicher als bei vorhandenen oder geeigneten Präventivmaßnahmen sind. Hierfür werden die folgenden Interaktionsvariablen definiert, die sich zu den bisherigen Interaktionsvariablen für die Corporate-GovernanceMechanismen und Qualitätsmerkmalen der Abschlussprüfungen invers oder gegenläufig verhalten: ${ }^{1616}$

1615 Analog Almaleeh, N. M. S. (2019), S. 36; McVay, S. E. (2006), S. 528.

1616 Mit den Alternativhypothesen $\mathrm{H}_{2.7}$ und mit $_{2.8}$ wurden für die Größen respektive Branchenspezialisierungen der Abschlussprüfer keine Wirkungen auf abschlusspolitische Ergebnisspaltungen postuliert. Im Folgenden wird untersucht, ob kleine bzw. mittelgroße oder branchendiversifizierte Abschlussprüfer abschlusspolitische Ergebnisspaltungen begünstigen. 
- AR_UW $I_{i, t}$ wird der Wert 1 zugewiesen, ${ }^{1617}$ falls für Aufsichtsräte variable Vergütungssysteme vorhanden sind ${ }^{1618}$ und kein Prüfungsausschuss existiert. Andernfalls erhält $A R_{-} U W I_{i, t}$ den Wert 0 .

- $V V E R G_{-} U W I_{i, t}=\frac{\text { Variable Vorstandsvergütung mit kurzfr. Bemessungsgrundlage } e_{i, t}}{\text { gesamte Vorstandsvergütung }} \cdot 100 \%$ misst die prozentualen Anteile der variablen Vorstandsvergütungen mit kurzfristigen Bemessungsgrundlagen an den gesamten Vorstandsvergütungen.

- $K_{-} G R A K_{i, t}$ wird der Wert 1 zugewiesen, ${ }^{1619}$ falls kein Aktionär mindestens fünf Prozent der Stimmrechte des Unternehmens zum Abschlussstichtag hält. Andernfalls erhält $K_{-} G R A K_{i, t}$ den Wert 0 .

- $E K Q_{i, t}=\frac{\text { Eigenkapital }_{i, t}}{\text { Bilanzsumme } F, i, t} \cdot 100 \%$ misst die Eigenkapitalquoten der fortgeführten Geschäftsbereiche in Prozent.

- $U E W W_{-} H_{-} P_{S, i, t}$ wird der Wert 1 zugewiesen, falls der Abschlussprüfer ein positives abnormales Prüfungshonorar erhält. Andernfalls erhält $U E W{ }_{-} P H_{-} P_{S, i, t}$ den Wert 0 .

- NPH_GH $H_{S, i, t}=\frac{\text { Nichtprüfungshonorar }_{S, i, t}}{\text { Gesamthonorar }_{S, i, t}} \cdot 100 \%$ misst den Anteil des Nichtprüfungshonorars am Gesamthonorar des Abschlussprüfers in Prozent.

- $K_{-} B 5_{i, t}$ wird der Wert 1 zugewiesen, falls weder BDO, Deloitte, EY, KPMG noch PwC der Abschlussprüfer ist. Andernfalls erhält $K_{-} B 5_{i, t}$ den Wert 0.

- $A P D I V_{i, t}=100 \%-A P S P E Z_{i, t}=100 \%-\frac{\sum_{i=1}^{N} \sqrt{\text { Bilanzsumme }_{S, i, t, k}}}{\sum_{k=1}^{K} \sum_{i=1}^{N} \sqrt{\text { Bilanzsumme }_{S, i, t}}} \cdot 100 \%$ misst die Branchendiversifizierungen der Abschlussprüfer.

Insgesamt sind die obigen nominalskalierten Interaktionsvariablen so definiert, dass beim Wert 1 die jeweiligen Präventivmaßnahmen nicht vorhanden sind. Analog sind die obigen verhältnisskalierten Interaktionsvariablen so definiert, dass die jeweilige Präventivmaßnahmen mit steigenden Werten an Wirkung verliert. In den Tab. 52 bis 55 werden die notwendige und hinreichende Bedingung für abschlusspolitische Ergebnisspaltungen zum Erhöhen der Ergebnisse aus den fortgeführten Geschäftsbereichen bei fehlenden oder ungeeigneten Präventivmaßnahmen geschätzt. In keinem dieser Szenarien ist sowohl die notwendige als auch die hinreichende Bedingung für abschlusspolitische Ergebnisspaltungen zum Erhöhen der Ergebnisses aus den fortgeführten Geschäftsbereichen erfüllt. Insofern scheinen fehlende oder ungeeignete Präventivmaß-

1617 Die Buchstabenfolge „UWI“ im Variablennamen $A R_{-} U W I_{i, t}$ bedeutet, dass Aufsichtsräte i. S.d. Alternativhypothese $\mathrm{H}_{2.1}$ unwirksam sind.

1618 Sitzungsgelder gelten bei der Variable $A R_{-} U W I_{i, t}$ nicht als variable Vergütungen.

1619 Der Buchstabe „, $K^{\prime \prime}$ im Variablennamen $K_{-} G R A K_{i, t}$ bedeutet, dass kein Großaktionär i. S. d. Alternativhypothese $\mathrm{H}_{2.3}$ vorhanden ist. 
nahmen abschlusspolitische Ergebnisspaltungen zum Erhöhen der Ergebnisse aus den fortgeführten Geschäftsbereichen nicht zu begünstigen und für Vorstände keine diesbezüglichen Anreize darzustellen. ${ }^{1620}$

\begin{tabular}{|c|c|c|c|c|c|}
\hline \multicolumn{2}{|c|}{ Regressionsgleichung (18a) } & \multicolumn{4}{|c|}{ Interaktionsvariable } \\
\hline$U E W_{-} B E_{F, i, t}$ & Koeff. & $A R_{-} U W I_{i, t}$ & $K_{-} G R A K_{i, t}$ & $U E W_{-} P H_{-} P_{S, i, t}$ & $K_{-} B 5_{i, t}$ \\
\hline$E A G B \_N_{i, t}$ & $\zeta_{1}$ & $\begin{array}{c}-1,2856 \\
(-5,77)^{* * *}\end{array}$ & $\begin{array}{c}-2,4571 \\
(-2,20)^{* *}\end{array}$ & $\begin{array}{c}-0,7730 \\
(-4,56)^{* * *}\end{array}$ & $\begin{array}{c}-1,3787 \\
(-6,20)^{* * *}\end{array}$ \\
\hline$A R_{-} U W I_{i, t}$ & $\zeta_{2}$ & $\begin{array}{l}0,0014 \\
(0,20)\end{array}$ & & & \\
\hline$K_{-} G R A K_{i, t}$ & $\zeta_{2}$ & & $\begin{array}{l}-0,0028 \\
(-0,25)\end{array}$ & & \\
\hline$U E W_{-} P H_{-} P_{S, i, t}$ & $\zeta_{2}$ & & & $\begin{array}{l}-0,0092 \\
(-1,36)\end{array}$ & \\
\hline$K_{-} B 5_{i, t}$ & $\zeta_{2}$ & & & & $\begin{array}{l}-0,0057 \\
(-0,72)\end{array}$ \\
\hline $\begin{array}{l}E A G B \_N_{i, t} \\
\cdot A R \_U W I_{i, t}\end{array}$ & $\zeta_{3}$ & $\begin{array}{c}0,3728 \\
(-0,35)\end{array}$ & & & \\
\hline$K_{-} G R A K_{i, t}$ & $\zeta_{3}$ & & $\begin{array}{l}1,3412 \\
(1,24)\end{array}$ & & \\
\hline$\cdot U E W W_{-} P H_{-} P_{S, i, t}$ & $\zeta_{3}$ & & & $\begin{array}{c}-0,8855 \\
(-3,49)^{* * *}\end{array}$ & \\
\hline$\cdot K_{-} B 5_{i, t}$ & $\zeta_{3}$ & & & & $\begin{array}{c}0,9136 \\
(2,58)^{* *}\end{array}$ \\
\hline Beob. & & 325 & 325 & 325 & 325 \\
\hline Adj. $R^{2}$ & & 0,2720 & 0,2795 & 0,2793 & 0,2743 \\
\hline \multicolumn{6}{|c|}{$\begin{array}{l}\text { Restliche Terme aus Regressionsgleichung (18a) nicht abgedruckt. t-Statistiken in Klammern. } \\
\text { Variablendefinitionen in Tab. 19. Obige Tests sind zweiseitig. }\end{array}$} \\
\hline $\mathrm{H}_{0}: \zeta_{1}+\zeta_{3} \geq 0$ & & $\begin{array}{l}-1,6584 \\
(-1,58)^{*}\end{array}$ & $\begin{array}{l}-1,1159 \\
(-5,56)^{* * *}\end{array}$ & $\begin{array}{l}-1,6585 \\
(-8,25)^{* * *}\end{array}$ & $\begin{array}{l}-0,4651 \\
(-1,75)^{* *}\end{array}$ \\
\hline
\end{tabular}

Tab. 52: Schätzung der notwendigen Bedingung für die Existenz von abschlusspolitischen Ergebnisspaltungen beim Erhöhen der Ergebnisse aus den fortgeführten Geschäftsbereichen bei fehlenden Präventivmaßnahmen mit nominalskalierten Interaktionsvariablen

1620 Auf die Untersuchung von abschlusspolitischen Ergebnisspaltungen bei fehlenden oder ungeeigneten Präventivmaßnahmen in Verbindung mit ineffizienten Unternehmensgrößen, beim Erreichen von Referenzwerten, bei Cookie Jars und bei Big Baths wird verzichtet. 


\begin{tabular}{|c|c|c|c|c|c|}
\hline \multicolumn{2}{|c|}{ Regressionsgleichung (18b) } & \multicolumn{4}{|c|}{ Interaktionsvariable } \\
\hline$U E W \_\Delta B E_{F, i, t+1}$ & Koeff. & $A R_{-} U W I_{i, t}$ & $K_{-} G R A K_{i, t}$ & $U E W_{-} P H_{-} P_{S, i, t}$ & $K_{-} B 5_{i, t}$ \\
\hline$E A G B_{-} N_{i, t}$ & $\zeta_{15}$ & $\begin{array}{c}-0,3635 \\
(-2,56)^{* *}\end{array}$ & $\begin{array}{l}0,3737 \\
(0,57)\end{array}$ & $\begin{array}{c}-0,5409 \\
(-2,89)^{* * *}\end{array}$ & $\begin{array}{l}-0,2693 \\
(-1,60)\end{array}$ \\
\hline$A R_{-} U W I_{i, t}$ & $\zeta_{16}$ & $\begin{array}{l}0,0035 \\
(0,38)\end{array}$ & & & \\
\hline$K_{-} G R A K_{i, t}$ & $\zeta_{16}$ & & $\begin{array}{l}0,0020 \\
(0,18)\end{array}$ & & \\
\hline$U E W \_P H \_P_{S, i, t}$ & $\zeta_{16}$ & & & $\begin{array}{l}-0,0041 \\
(-0,48)\end{array}$ & \\
\hline$K_{-} B 5_{i, t}$ & $\zeta_{16}$ & & & & $\begin{array}{l}-0,0047 \\
(-0,49)\end{array}$ \\
\hline $\begin{array}{l}E A G B \_N_{i, t} \\
\cdot A R \_U W I_{i, t}\end{array}$ & $\zeta_{17}$ & $\begin{array}{l}0,4933 \\
(0,63)\end{array}$ & & & \\
\hline$K_{-} G R A K_{i, t}$ & $\zeta_{17}$ & & $\begin{array}{l}-0,8016 \\
(-1,21)\end{array}$ & & \\
\hline$\cdot U E W_{-} P H_{-} P_{S, i, t}$ & $\zeta_{17}$ & & & $\begin{array}{l}0,3788 \\
(1,42)\end{array}$ & \\
\hline$\cdot K_{-} B 5_{i, t}$ & $\zeta_{17}$ & & & & $\begin{array}{c}-0,6074 \\
(-2,33)^{* *}\end{array}$ \\
\hline$E A G B_{-} N_{i, t+1}$ & $\zeta_{18}$ & $\begin{array}{l}-1,0212 \\
(-5,20)^{* * *}\end{array}$ & $\begin{array}{c}-1,0998 \\
(-6,00)^{* * *}\end{array}$ & $\begin{array}{c}-1,0563 \\
(-5,10)^{* * *}\end{array}$ & $\begin{array}{l}-1,0246 \\
(-5,10)^{* * *}\end{array}$ \\
\hline $\begin{array}{l}\text { Beob. } \\
\text { Adj. } \mathrm{R}^{2}\end{array}$ & & $\begin{array}{l}325 \\
-0,0007\end{array}$ & $\begin{array}{l}325 \\
0,0014\end{array}$ & $\begin{array}{l}325 \\
0,0003\end{array}$ & $\begin{array}{l}325 \\
-0,0004\end{array}$ \\
\hline
\end{tabular}

Restliche Terme aus Regressionsgleichung (18b) nicht abgedruckt. t-Statistiken in Klammern. Variablendefinitionen in Tab. 19. Obige Tests sind zweiseitig.

\begin{tabular}{lcllc}
\hline $\mathrm{H}_{0}: \zeta_{15}+\zeta_{17} \leq 0$ & 0,1298 & $-0,4279$ & $-0,1620$ & $-0,8767$ \\
& $(0,17)$ & $(-3,45)$ & $(-0,80)$ & $(-4,43)$ \\
\hline
\end{tabular}

Tab. 53: Schätzung der hinreichenden Bedingung für die Existenz von abschlusspolitischen Ergebnisspaltungen beim Erhöhen der Ergebnisse aus den fortgeführten Geschäftsbereichen bei fehlenden Präventivmaßnahmen mit nominalskalierten Interaktionsvariablen 


\begin{tabular}{|c|c|c|c|c|c|}
\hline \multicolumn{2}{|c|}{ Regressionsgleichung (18a) } & \multicolumn{4}{|c|}{ Interaktionsvariable } \\
\hline$U E W_{-} B E_{F, i, t}$ & Koeff. & $\overline{V V E R G \_U W I_{i, t}}$ & $E K Q_{i, t}$ & $N P H_{-} G H_{S, i, t}$ & $A P D I V_{i, t}$ \\
\hline$E A G B_{-} N_{i, t}$ & $\zeta_{1}$ & $\begin{array}{l}-0,7612 \\
(-1,50)\end{array}$ & $\begin{array}{l}-1,7324 \\
(-7,71)^{* * *}\end{array}$ & $\begin{array}{c}-1,6500 \\
(-17,62)^{* * *}\end{array}$ & $\begin{array}{l}0,1715 \\
(0,18)\end{array}$ \\
\hline$V V E R G_{-} U W I_{i, t}$ & $\zeta_{2}$ & $\begin{array}{l}-0,0002 \\
(-0,61)\end{array}$ & & & \\
\hline$E K Q_{i, t}$ & $\zeta_{2}$ & & $\begin{array}{l}-0,0000 \\
(-0,12)\end{array}$ & & \\
\hline$N P H_{-} G H_{S, i, t}$ & $\zeta_{2}$ & & & $\begin{array}{l}0,0146 \\
(0,90)\end{array}$ & \\
\hline$A P D I V_{i, t}$ & $\zeta_{2}$ & & & & $\begin{array}{l}0,0001 \\
(0,35)\end{array}$ \\
\hline $\begin{array}{l}E A G B N_{i, t} \\
\cdot V V E R G_{-} U W I_{i, t}\end{array}$ & $\zeta_{3}$ & $\begin{array}{l}-0,0338 \\
(-1,14)\end{array}$ & & & \\
\hline$\cdot E K Q_{i, t}$ & $\zeta_{3}$ & & $\begin{array}{r}0,0136 \\
(1,96)^{*}\end{array}$ & & \\
\hline$\cdot N P H_{-} G H_{S, i, t}$ & $\zeta_{3}$ & & & $\begin{array}{c}1,4332 \\
(2,56)^{* *}\end{array}$ & \\
\hline$\cdot A P D I V_{i, t}$ & $\zeta_{3}$ & & & & $\begin{array}{l}-0,0186 \\
(-1,52)\end{array}$ \\
\hline Beob. & & 325 & 325 & 325 & 325 \\
\hline Adj. $R^{2}$ & & 0,2768 & 0,2744 & 0,2750 & 0,2741 \\
\hline
\end{tabular}

Restliche Terme aus Regressionsgleichung (18a) nicht abgedruckt. t-Statistiken in Klammern. Variablendefinitionen in Tab. 19. Obige Tests sind zweiseitig.

(1) $\mathrm{H}_{0}: \zeta_{1}+\zeta_{3}\left\{I N C_{-} 1\right\}_{N} \geq 0$

$$
\begin{array}{cccc}
-1,0915 & -1,2870 & -1,6010 & -1,0498 \\
(-4,38)^{* * *} & (-8,58)^{* * *} & (-18,35)^{* * *} & (-4,91)^{* * *}
\end{array}
$$

(2) $\mathrm{H}_{0}: \zeta_{1}+\zeta_{3}\left\{I N C_{-} l\right\}_{H} \geq 0$

$$
\begin{array}{llcc}
-2,3664 & -0,7110 & -1,0408 & -1,6878 \\
(-2,54)^{* * *} & (-1,91)^{* *} & (-4,84)^{* * *} & (-5,80)^{* * *}
\end{array}
$$

(3) $\mathrm{H}_{0}: \zeta_{3}\left(\left\{I N C_{-} 1\right\}_{H}-\left\{I N C_{-} l\right\}_{N}\right) \geq 0$

\begin{tabular}{cccc}
$-1,2749$ & 0,5760 & 0,5602 & $-0,6380$ \\
$(-1,14)$ & $(1,96)$ & $(2,56)$ & $(-1,52)^{*}$ \\
\hline$\left\{I N C_{-} l\right\}_{N}=\left\{V V E R G \_U W I_{i, t, N}, E K Q_{i, t, N}, N P H_{-} G H_{S, i, t, N}, A P D I V_{i, t, N}\right\}$ \\
$\left\{I N C_{-} l\right\}_{H}=\left\{V V E R G_{-} U W I_{i, t, H}, E K Q_{i, t, H}, N P H_{-} G H_{S, i, t, H}, A P D I V_{i, t, H}\right\}$
\end{tabular}

Tab. 54: Schätzung der notwendigen Bedingung für die Existenz von abschlusspolitischen Ergebnisspaltungen beim Erhöhen der Ergebnisse aus den fortgeführten Geschäftsbereichen bei fehlenden Präventivmaßnahmen mit verhältnisskalierten Interaktionsvariablen 


\begin{tabular}{|c|c|c|c|c|c|}
\hline \multicolumn{2}{|c|}{ Regressionsgleichung (18b) } & \multicolumn{4}{|c|}{ Interaktionsvariable } \\
\hline$U E W \_\Delta B E_{F, i, t+1}$ & Koeff. & $V V E R G_{-} U W I_{i, t}$ & $E K Q_{i, t}$ & $N P H_{-} G H_{S, i, t}$ & $A P D I V_{i, t}$ \\
\hline$E A G B_{-} N_{i, t}$ & $\zeta_{15}$ & $\begin{array}{l}-0,1627 \\
(-0,41)\end{array}$ & $\begin{array}{l}-0,0284 \\
(-0,12)\end{array}$ & $\begin{array}{l}-0,0821 \\
(-0,56)\end{array}$ & $\begin{array}{l}-0,6936 \\
(-0,77)\end{array}$ \\
\hline$V V E R G_{-} U W I_{i, t}$ & $\zeta_{16}$ & $\begin{array}{l}-0,0005 \\
(-1,81)^{*}\end{array}$ & & & \\
\hline$E K Q_{i, t}$ & $\zeta_{16}$ & & $\begin{array}{l}-0,0002 \\
(-0,57)\end{array}$ & & \\
\hline$N P H_{-} G H_{S, i, t}$ & $\zeta_{16}$ & & & $\begin{array}{l}-0,0307 \\
(-1,57)\end{array}$ & \\
\hline$A P D I V_{i, t}$ & $\zeta_{16}$ & & & & $\begin{array}{l}-0,0004 \\
(-1,44)\end{array}$ \\
\hline $\begin{array}{l}E A G B \_N_{i, t} \\
\cdot V V E R G_{-} U W I_{i, t}\end{array}$ & $\zeta_{17}$ & $\begin{array}{l}-0,0056 \\
(-0,31)\end{array}$ & & & \\
\hline$\cdot E K Q_{i, t}$ & $\zeta_{17}$ & & $\begin{array}{l}-0,0089 \\
(-1,44)\end{array}$ & & \\
\hline$\cdot N P H_{-} G H_{S, i, t}$ & $\zeta_{17}$ & & & $\begin{array}{l}-1,0152 \\
(-1,93)^{*}\end{array}$ & \\
\hline$\cdot A P D I V_{i, t}$ & $\zeta_{17}$ & & & & $\begin{array}{l}0,0047 \\
(0,44)\end{array}$ \\
\hline$E A G B \_N_{i, t+1}$ & $\zeta_{18}$ & $\begin{array}{l}-1,0396 \\
(-5,29)^{* * *}\end{array}$ & $\begin{array}{l}-1,0219 \\
(-4,73)^{* * *}\end{array}$ & $\begin{array}{l}-1,0436 \\
(-4,89)^{* * *} \\
\end{array}$ & $\begin{array}{l}-1,0339 \\
(-5,67)^{* * *}\end{array}$ \\
\hline $\begin{array}{l}\text { Beob. } \\
\text { Adj. } R^{2}\end{array}$ & & $\begin{array}{l}325 \\
0,0083\end{array}$ & $\begin{array}{l}325 \\
0,0005\end{array}$ & $\begin{array}{l}325 \\
0,0052\end{array}$ & $\begin{array}{l}325 \\
0,0071\end{array}$ \\
\hline
\end{tabular}

Restliche Terme aus Regressionsgleichung (18b) nicht abgedruckt. t-Statistiken in Klammern. Variablendefinitionen in Tab. 19. Obige Tests sind zweiseitig.

(1) $\mathrm{H}_{0}: \zeta_{15}+\zeta_{17}\left\{I N C_{-} l\right\}_{N} \leq 0$

$$
\begin{array}{llll}
-0,2179 & -0,3205 & -0,1168 & -0,3836 \\
(-0,94) & (-2,18) & (-0,84) & (-1,66)
\end{array}
$$

(2) $\mathrm{H}_{0}: \zeta_{15}+\zeta_{17}\left\{I N C_{-} 1\right\}_{H} \leq 0$

(3) $\mathrm{H}_{0}: \zeta_{17}\left(\left\{I N C_{-} 1\right\}_{H}-\left\{I N C_{-} 1\right\}_{N}\right) \leq 0$

$\begin{array}{llll}-0,4308 & -0,6983 & -0,5136 & -0,2217 \\ (-0,85) & (-2,17) & (-2,65) & (-0,99)\end{array}$

\begin{tabular}{cccc}
$-0,2129$ & $-0,3778$ & $-0,3968$ & 0,1619 \\
$(-0,31)$ & $(-1,44)$ & $(-1,93)$ & $(0,44)$ \\
\hline
\end{tabular}

$\left\{I N C_{-}\right\}_{N}=\left\{V V E R G_{-} U W I_{i, t, N}, E K Q_{i, t, N}, N P H_{-} G H_{S, i, t, N}, A P D I V_{i, t, N}\right\}$ $\left\{I N C_{-}\right\}_{H}=\left\{V V E R G_{-}^{-} U W I_{i, t, H}, E K Q_{i, t, H}, N P H_{-} G H_{S, i, t, H}, A P D I V_{i, t, H}\right\}$

Tab. 55: Schätzung der hinreichenden Bedingung für die Existenz von abschlusspolitischen Ergebnisspaltungen beim Erhöhen der Ergebnisse aus den fortgeführten Geschäftsbereichen bei fehlenden Präventivmaßnahmen mit verhältnisskalierten Interaktionsvariablen 
(4) Die Existenz von aufgegebenen Geschäftsbereichen erhöht die Komplexität der Abschlussprüfungen, da Abschlussprüfer bspw. die damit verbundenen Anhangangaben, etwaige Änderungen der rechtlichen Strukturen der Geschäftsbereiche sowie den getrennten Ausweis der aufgegebenen Geschäftsbereiche in den Bilanzen und GuV prüfen müssen. Aufgrund dieser zusätzlichen Komplexität könnten Abschlussprüfer höhere Prüfungshonorare fordern. In der Tab. 37 wurden logarithmierte Prüfungshonorare mit zahlreichen Determinanten geschätzt, die in der Fachliteratur weit verbreitet sind. Dem Verfasser der vorliegenden Arbeit ist jedoch keine Studie bekannt, die untersucht, ob die Existenz von aufgegebenen Geschäftsbereichen die Höhe der Prüfungshonorare beeinflusst. Daher ist in der Schätzung der logarithmierten Prüfungshonorare in der Tab. 56 die Variable $E A G B_{-} E_{i, t}$ enthalten, die bereits in der ergänzenden Analyse (2) definiert wurde. Allerdings ist die Variable $E A G B_{-} E_{i, t}$ in der Tab. 56 statistisch nicht signifikant, weshalb aufgegebene Geschäftsbereiche nicht mit höheren Prüfungshonoraren verbunden sind. Verkleinern sich die Konsolidierungskreise, nachdem aufgegebene Geschäftsbereiche verkauft oder stillgelegt wurden, könnten die Prüfungshonorare in den Folgeperioden sogar sinken.

\begin{tabular}{lrrcrrr}
\hline$L N P H_{S, i, t}$ & \multicolumn{1}{c}{ Koeff. } & Std.fehl. & t-Stat. & p-Wert & Unt. KI & Ob. KI \\
\hline EAGB_E $E_{i, t}$ & 0,0744 & 0,1374 & 0,5416 & 0,5897 & $-0,1993$ & 0,3481 \\
$L N B S_{S, i, t}$ & 0,5781 & 0,0546 & $10,5930^{* * *}$ & 0,0000 & 0,4694 & 0,6868 \\
$J F \_V J_{S, i, t}$ & 0,2529 & 0,0619 & $4,0838^{* * *}$ & 0,0001 & 0,1296 & 0,3763 \\
$W \_V S G_{S, i, t}$ & 0,0071 & 0,0044 & 1,6194 & 0,1095 & $-0,0016$ & 0,0158 \\
$Z S C R_{S, i, t}$ & 0,0000 & 0,0000 & 1,3982 & 0,1661 & $-0,0000$ & 0,0000 \\
$W \_U E_{S, i, t}$ & $-0,0016$ & 0,0013 & $-1,2195$ & 0,2264 & $-0,0041$ & 0,0010 \\
$B 5_{i, t}$ & 0,0799 & 0,1029 & 0,7764 & 0,4399 & $-0,1250$ & 0,2848 \\
$N P H_{-} P H_{S, i, t}$ & $-0,1606$ & 0,0447 & $-3,5954^{* * *}$ & 0,0006 & $-0,2496$ & $-0,0717$ \\
$D A X_{i, t}$ & 0,7086 & 0,3927 & $1,8046^{*}$ & 0,0751 & $-0,0734$ & 1,4907 \\
$M D A X_{i, t}$ & 0,7566 & 0,2370 & $3,1926^{* * *}$ & 0,0021 & 0,2846 & 1,2287 \\
$S D A X_{i, t}$ & 0,3430 & 0,2662 & 1,2884 & 0,2015 & $-0,1872$ & 0,8733 \\
$T A X_{i, t}$ & 0,1380 & 0,1203 & 1,1475 & 0,2548 & $-0,1015$ & 0,3775 \\
$J_{2009, i, t}$ & $-0,0238$ & 0,0473 & $-0,5034$ & 0,6161 & $-0,1180$ & 0,0704 \\
$J_{2010, i, t}$ & $-0,0082$ & 0,0513 & $-0,1605$ & 0,8729 & $-0,1104$ & 0,0939 \\
$J_{2011, i, t}$ & $-0,0424$ & 0,0633 & $-0,6705$ & 0,5046 & $-0,1685$ & 0,0836 \\
$J_{2012, i, t}$ & $-0,0514$ & 0,0666 & $-0,7710$ & 0,4431 & $-0,1841$ & 0,0813 \\
$J_{2013, i, t}$ & $-0,0444$ & 0,0684 & $-0,6494$ & 0,5180 & $-0,1806$ & 0,0918 \\
Konstante & $-1,6546$ & 0,5888 & $-2,8100^{* * *}$ & 0,0063 & $-2,8272$ & $-0,4819$ \\
\hline Beob. & 325 & & & & & \\
Adj. R ${ }^{2}$ & 0,8446 & & & & & \\
\hline Variablendefinitionen in Tab. 19. Ausschließlich zweiseitige Tests. & \\
\hline
\end{tabular}

Tab. 56: Schätzung der logarithmierten Prüfungshonorare mit aufgegebenen Geschäftsbereichen als zusätzliche Determinante 


\subsection{Grenzen}

Neben den allgemeinen Irrtumswahrscheinlichkeiten, die bei statistischen Tests akzeptiert werden, ${ }^{1621}$ unterliegt die empirische Untersuchung in der vorliegenden Arbeit zahlreichen weiteren Einschränkungen. Die wichtigsten dieser Einschränkungen werden im Folgenden beleuchtet:

(1) Die Grundgesamtheit für die empirische Untersuchung bilden 895 Beobachtungen von Technologieunternehmen. Die Grundgesamtheit der vorliegenden Arbeit wurde auf die Technologiebranche beschränkt, da sie im CDAX in den Jahren 2007 bis 2014 die meisten Mitglieder hat und die Technologiebranche aufgrund ihrer hohen Forschungsund Entwicklungsintensität technische und wirtschaftliche Misserfolge und somit aufgegebene Geschäftsbereiche begünstigt. Trotz dieser günstigen Voraussetzungen müssen aus verschiedenen Gründen 570 Beobachtungen ausgeschlossen werden, sodass für die Stichprobe lediglich 325 Beobachtungen verbleiben, von denen nur 42 Beobachtungen oder knapp 13 Prozent über aufgegebene Geschäftsbereiche verfügen. ${ }^{1622}$ Aufgrund dieser geringen Anzahl an Beobachtungen aus der Technologiebranche wurden kleinere Branchen nicht ausgewertet, obwohl die Untersuchung durch die Auswertung von anderen Branchen deutlich aussagekräftiger werden würde.

(2) Mit dem oben erwähnten Ausschluss von 570 Beobachtungen gehen sehr viele Informationen verloren. Kritischer als dieser allgemeine Informationsverlust ist jedoch, dass die Ausschlüsse eine sog. Selection Bias bzw. Auswahlverzerrung verursachen können. ${ }^{1623}$ Um die potenzielle Auswahlverzerrung erkennen zu können, ist es hilfreich, die Gründe zu betrachten, die besonders viele Ausschlüsse nach sich ziehen. Neben Insolvenzen zählen hierzu Unternehmen, von denen keine Jahres- bzw. Konzernabschlüsse aus mindestens drei aufeinanderfolgenden Geschäftsjahren vorliegen. ${ }^{1624}$ Diese Ausschlüsse betreffen vor allem finanziell instabile Unternehmen, ${ }^{1625}$ welche die mit CDAX-Notierungen verbundenen Anforderungen möglicherweise nicht mehr erfüllen können und sich aus dem CDAX zurückziehen oder aufgrund von administrativen Problemen keine Jahres- bzw. Konzernabschlüsse mehr veröffentlichen. Ebenso sind finanziell angeschlagene Unternehmen typische Übernahmekandidaten, wodurch sie ebenfalls aus dem CDAX ausscheiden können. Im Ergebnis verbleiben durch die

1621 Hierzu Bortz, J./Döring, N. (2006), S. 22 f./26-28; Ruhnke, K. (2000), S. 233, Fn. 5.

1622 Von Seve, F. (2016), S. 210 wird bereits eine Stichprobe mit 2.186 Beobachtungen, in der 285 Beobachtungen aufgegebene Geschäftsbereiche ausweisen, als klein bezeichnet.

1623 Weiterführend Wooldridge, J. M. (2020), S. 316/586-592.

1624 Siehe die Tab. 12. Einen weiteren wichtigen Ausschlussgrund bilden Beobachtungen von Unternehmen, von denen zwar Jahres- bzw. Konzernabschlüsse aus mindestens drei aufeinanderfolgenden Geschäftsjahren vorliegen, aber die das erste oder letzte Glied dieser Sequenz bilden. Die Informationen aus diesen Beobachtungen gehen im Rahmen von zeitverschobenen Variablen zwar in das ökonometrische Modell ein, werden in der Stichprobe aber nicht mitgezählt. Siehe hierzu den Abschnitt 8.1.

1625 Analog Köke, J. (2004), S. 59 f. 
Ausschlüsse von insolventen Unternehmen und von Unternehmen, von denen keine Jahres- bzw. Konzernabschlüsse aus mindestens drei aufeinanderfolgenden Geschäftsjahren vorliegen, vor allem finanziell stabile Unternehmen in der Stichprobe. Wenn sich finanziell stabile Unternehmen bei Ergebnisspaltungen mit aufgegebenen Geschäftsbereichen systematisch anders verhalten als finanziell angeschlagene Unternehmen, kann die Auswahlverzerrung auch die Testergebnisse verzerren. Beispielsweise können finanziell angeschlagene Unternehmen zu abschlusspolitischen Ergebnisspaltungen neigen, um ihre finanziellen Probleme zu verbergen, wohingegen finanziell stabile Unternehmen auf abschlusspolitische Ergebnisspaltungen und die damit verbundenen Reputationsrisiken verzichten. Sind in der Stichprobe finanziell stabile Unternehmen überrepräsentiert, werden dort evtl. keine abschlusspolitischen Ergebnisspaltungen festgestellt, obwohl sie in der Grundgesamtheit möglicherweise auftreten. Diese Auswahlverzerrung, die aus der Voraussetzung einer CDAX-Notierung in mindestens drei aufeinanderfolgenden Geschäftsjahren resultiert, ist naheliegend, lässt sich für die vorliegende Untersuchung aber nicht vermeiden. Schließlich können aus dem CDAX ausgeschiedene Unternehmen auf eine IFRS-Rechnungslegung verzichten und sind dann bezüglich abschlusspolitischen Ergebnisspaltungen unter IFRS 5 nicht mehr relevant.

(3) Die Aussagekraft der gesamten multiplen Analyse und insbesondere der Hypothesentests hängt stark davon ab, wie zuverlässig die bereinigten Betriebsergebnismargen anhand der Regressionsgleichung (7) geschätzt werden können. Die darauf aufbauende Gleichung (8) für die erwarteten bereinigten Betriebsergebnismargen erhebt den Anspruch, die bereinigten Betriebsergebnismargen in denjenigen Höhe berechnen zu können, in denen sie ohne abschlusspolitische Maßnahmen vorliegen würden. Die implizite Annahme hinter diesem Vorgehen lautet, dass die meisten Unternehmen die bereinigte Betriebsergebnismargen nicht abschlusspolitisch beeinflussen und es somit auffällt, wenn einzelne Unternehmen hiervon abweichen. Anders ausgedrückt suggeriert die vorliegende Arbeit exakte Berechnungen der bereinigten Betriebsergebnismargen ohne abschlusspolitische Maßnahmen, obwohl die hierfür erforderlichen unternehmensinternen Daten nicht vorliegen. Können die bereinigten Betriebsergebnismargen ohne abschlusspolitische Maßnahmen nicht ausreichend genau geschätzt werden, sind die multiplen Analysen und Hypothesentests nicht aussagekräftig oder sogar irreführend. Diese Einschränkung gilt analog für die Differenzen der bereinigten Betriebsergebnismargen, die in der Regressionsgleichung (10) und in der Tab. 24 geschätzt werden. Diese Einschränkung lässt sich nur dadurch etwas relativieren, dass die adjustierten Bestimmtheitsmaße mit rund 0,7 in der Tab. 22 und mit rund 0,6 in der Tab. 24 angemessene Modellgüten vermuten lassen. 
(4) Unerwartet hohe oder niedrige bereinigte Betriebsergebnismargen müssen nicht durch abschlusspolitische Ergebnisspaltungen begründet sein, sondern können aus strategischen Vor- oder Nachteilen resultieren. ${ }^{1626}$ In der vorliegenden Arbeit werden unternehmensstrategische Aspekte jedoch nur beiläufig betrachtet, ${ }^{1627}$ da die Rechnungslegung und Corporate Governance einschließlich den Abschlussprüfungen im Fokus stehen. Verfügen Unternehmen aber über strategische Vor- oder Nachteile und gleichzeitig aufgebene Geschäftsbereiche, kann zwischen den unerwarteten bereinigten Betriebsergebnismargen und den Ergebnismargen aus den aufgegebenen Geschäftsbereichen eine Korrelation entstehen, die irrtümlich abschlusspolitische Ergebnisspaltungen suggeriert. Diese Einschränkung lässt sich zunächst dadurch relativieren, dass bei der Schätzung der bereinigten Betriebsergebnismargen und bei der Messung des Zusammenhangs zwischen den unerwarteten bereinigten Betriebsergebnismargen und den Ergebnissen aus den aufgegebenen Geschäftsbereichen einige Variablen verwendet werden, die strategische Aspekte zumindest rudimentär abbilden können. ${ }^{1628} \mathrm{Zu}$ dem wird die Gefahr der Verwechslung von strategischen Vor- oder Nachteilen mit abschlusspolitischen Ergebnisspaltungen tendenziell durch die hinreichende Bedingung für die Feststellung von abschlusspolitischen Ergebnisspaltungen eingedämmt. Mit dieser hinreichenden Bedingung wird der Fortbestand der bereinigten Betriebsergebnismargen im Geschäftsjahr nach dem Abgang der Geschäftsbereiche getestet. Da strategische Vor- oder Nachteilen typischerweise einige Zeit beibehalten werden, ${ }^{1629}$ sollten die daraus resultierenden unerwarteten bereinigten Betriebsergebnismargen tendenziell persistent sein und von lediglich temporär wirksamen abschlusspolitischen Ergebnisspaltungen unterschieden werden können.

(5) Als notwendige Bedingung für den empirischen Nachweis von abschlusspolitischen Ergebnisspaltung wird untersucht, ob unerwartet hohe bereinigte Betriebsergebnismargen durch geringe Ergebnismargen aus den aufgegebenen Geschäftsbereichen erklärt werden können. ${ }^{1630}$ Während die bereinigten ordentlichen Betriebsergebnismar-

1626 Die Entstehung von strategischen Vorteilen diskutieren bspw. Argyres, N. S./Zenger, T. R. (2012), S. 1648; Barney, J. B. (1997), S. 146-160; Pitelis, C. N./Pseiridis, A. N. (1999), S. 229 mit weiteren Nennungen; Rumelt, R. P. (1984), S. 566 f.

1627 Siehe exemplarisch den Abschnitt 9.1 und S. 273.

1628 Siehe bei der Schätzung der bereinigten Betriebsergebnismargen im Abschnitt 8.5 bspw. die Erläuterungen der Variablen $U B V_{F, i, t}, W \_U E_{F, i, t}$ und $W_{-} U E_{-} N_{F, i, t}$ sowie bei der Messung des $\mathrm{Zu}-$ sammenhangs zwischen den unerwarteten bereinigten Betriebsergebnismargen und den Ergebnismargen aus den aufgegebenen Geschäftsbereichen im Abschnitt 9.1 bspw. die Erläuterung der Variable $G K R_{F, i, t}$.

1629 Vgl. Foss, N. J. (1997), S. 4.

1630 Genauer formuliert wird als notwendige Bedingung für abschlusspolitische Ergebnisspaltungen beim Erhöhen der Ergebnisse aus den fortgeführten Geschäftsbereichen, bei ineffizienten Unternehmensgrößen und beim Erreichen von Referenzwerten getestet, ob unerwartet hohe bereinigte Betriebsergebnismargen durch geringe Ergebnismargen aus den aufgegebenen Geschäftsbereichen erklärt werden können. Dagegen werden für abschlusspolitische Ergebnisspaltungen zwecks Cookie Jars und Big Baths analysiert, ob unerwartet niedrige bereinigte Betriebsergebnismargen durch hohe Ergebnismargen aus den aufgegebenen Geschäftsbereichen erklärt werden können. 
gen aus den fortgeführten Geschäftsbereichen um zahlreiche betriebsfremde und aperiodische Bestandteile bereinigt sind, ${ }^{1631}$ können die Ergebnismargen aus den aufgegebenen Geschäftsbereichen nicht entsprechend bereinigt werden, da diese nur in Umsatzerlöse, Aufwendungen und die Ergebnisse vor Steuern aufgegliedert werden müssen. ${ }^{1632}$ Insofern können die Ergebnismargen aus den aufgegebenen Geschäftsbereichen als unabhängige Variable tendenziell alleine aufgrund der enthaltenen betriebsfremden und aperiodischen Komponenten volatiler als die bereinigten Betriebsergebnismargen aus den fortgeführten Geschäftsbereichen als abhängige Variable sein. Diese asymmetrische Bereinigungen der Ertragsgrößen können einerseits die Korrelation zwischen den beiden Variablen verstärken und zu irrtümlich angenommenen Alternativhypothesen führen. Andererseits kann die höhere Volatilität der unabhängigen Variable eine tatsächlich vorliegende Korrelation überdecken oder sogar umkehren, wodurch die überwiegend gerichtet formulierten Alternativhypothesen irrtümlich abgelehnt werden. Für die hinreichende Bedingung der empirischen Feststellung von abschlusspolitischen Ergebnisspaltungen gilt die oben beschriebene Einschränkung ana$\log$.

(6) Das ökonometrische Modell kann abschlusspolitische Ergebnisspaltungen nur für regelmäßig anfallende Aufwendungen und Erträge der ordentlichen Betriebstätigkeit exklusive der Abschreibungen erfassen. ${ }^{1633}$ Werden planmäßige Abschreibungen ${ }^{1634}$ sowie außerordentliche Aufwendungen und Erträge abschlusspolitisch auf die aufgegebenen und fortgeführten Geschäftsbereiche verteilt, kann das vorliegende Modell diese abschlusspolitischen Ergebnisspaltungen nicht erfassen. Somit können abschlusspolitische Ergebnisspaltungen in der Realität ausgeprägter sein als diese in der vorliegenden Arbeit gemessen werden.

(7) Unabhängige Variablen werden endogen genannt und verzerren die Schätzungen der Regressionskoeffizienten, wenn die unabhängigen Variablen mit den Residuen korrelieren. ${ }^{1635}$ Endogenität kann drei Ursachen haben: (a) Eine unabhängige Variable ist falsch gemessen, (b) es fehlt eine wichtige unabhängige Variable oder (c) die abhängige und mindestens eine unabhängige Variable werden simultan bestimmt: ${ }^{1636}$

(a) Messfehler können in der vorliegenden Arbeit weitestgehend ausgeschlossen werden, da alle Variablen, die sich aus Jahres- bzw. Konzernabschlüssen entneh-

1631 Siehe die Definition der bereinigten ordentlichen Betriebsergebnismarge im Abschnitt 8.5, die an die Schemata in der Tab. 13 und der Tab. 14 anknüpft.

1632 Vgl. IFRS 5.33 Buchst. (b) Ziff. (i). Weiterführend Darrough, M./Lee, Y. G./Oh, H. I. (2019), S. 202, Fn 16; Moses, O. D. (1987), S. 360.

1633 Siehe hierzu die Fn. 1420.

1634 Im Abschnitt 8.5 ist ausführlich begründet, warum Abschreibungen nicht in den bereinigten Betriebsergebnismargen enthalten sind.

1635 Vgl. Wooldridge, J. M. (2020), S. 82 f./201/245/294/450/800.

1636 So Wooldridge, J. M. (2020), S. 534. 
men lassen, manuell erhoben wurden und deren Plausibilität mehrfach überprüft wurde. Die hohe Datengenauigkeit zeigt sich bspw. bei der Berechnung der ordentlichen Betriebsergebnisse in der Tab. 13 und in der Tab. 14, bei der betriebsfremde und aperiodische Bestandteile aus den ordentlichen Betriebsergebnissen deutlich differenzierter herausgefiltert werden als aus den Core Earnings, ${ }^{1637}$ die relativ pauschal nur außerordentliche Aufwendungen und Erträge ausschließen. Zudem zeigt sich die hohe Datenqualität darin, dass in der vorliegenden Arbeit nahezu alle Minima und Maxima aus der Tab. 20 betrachtet und auf die publizierten Zahlen in den Jahres- bzw. Konzernabschlüssen zurückgeführt werden können. ${ }^{1638}$

(b) Fehlende wichtige unabhängige Variablen können im Gegensatz zu Messfehlern nicht weitestgehend ausgeschlossen werden. ${ }^{1639}$ In der vorliegenden Arbeit wird bspw. postuliert, dass interne Corporate-Governance-Mechanismen abschlusspolitische Ergebnisspaltungen verhindern. In der Realität können Corporate-Governance-Mechanismen jedoch mit zunehmender Dauer seit ihren erfolgreichen Implementierungen wirksamer werden. So kann es einige Zeit dauern, bis neu eingeführte anreizorientierte Vergütungssysteme die Vorstände disziplinieren. In der vorliegenden Arbeit wird zwischen Unternehmen, die anreizorientierte Vergütungssysteme neu eingeführt haben, und Unternehmen, die seit vielen Jahren über anreizorientierte Vergütungssysteme verfügen, aber nicht unterschieden obwohl es bei letzteren wahrscheinlicher ist, dass abschlusspolitische Ergebnisspaltungen verhindert werden. Korreliert die fehlende unabhängige Variable für die Dauer seit den erfolgreichen Implementierungen der Corporate-GovernanceMechanismen mit einer der unabhängigen Variablen in der Regressionsgleichung, werden die Regressionskoeffizienten verzerrt geschätzt. ${ }^{1640}$ Mögliche fehlende Variablen sind die Unternehmensgrößen und die operativen Periodenabgrenzungen. ${ }^{1641}$ Die Unternehmensgrößen können mit der Dauer seit den erfolgreichen Implementierungen von Corporate-Governance-Mechanismen positiv korrelieren, da Unternehmen mit steigenden Größen durch die Kapitalmärkte und die Politik typischerweise nachdrücklicher zu einer professionellen Corporate Governance aufgefordert werden. Die operativen Periodenabgrenzungen können mit der Dauer seit den erfolgreichen Implementierungen negativ korrelieren, da die operativen Periodenabgrenzungen abschlusspolitische Bestandteile umfassen können, die durch Corporate-Governance-Mechanismen möglicherweise gesenkt werden. Diese Endogenität aufgrund einer fehlenden unabhängigen Variable kann

1637 Siehe die Gleichung (6).

1638 Hierzu allgemein Wooldridge, J. M. (2020), S. 317.

1639 Allgemein hierzu Bortz, J./Döring, N. (2006), S. 13 f.

1640 Siehe Frost, J. (o. J.), o. S.

1641 Siehe exemplarisch die unabhängigen Variablen $L N B S_{F, i, t}$ und $P A B_{F, i, t}$ in der Regressionsgleichung (15). 
vermieden werden, wenn die Dauer seit den erfolgreichen Implementierungen von Corporate-Governance-Mechanismen als zusätzliche unabhängige Variable in die Regressionsgleichung aufgenommen werden würden. Allerdings lässt sich diese Dauer oftmals nicht zuverlässig messen, weshalb sie nicht als unabhängige Variable berücksichtigt wird und die Gefahr einer Endogenität besteht.

(c) Simultane Bestimmungen der abhängigen und mindestens einer unabhängigen Variable können bspw. bei den Prüfungshonoraren der Abschlussprüfer und den unerwarteten bereinigen Betriebsergebnismargen auftreten. Vermuten Abschlussprüfer aufgrund von hohen unerwarteten bereinigen Betriebsergebnismargen abschlusspolitische Ergebnisspaltungen, können sie unter Verweis auf den erhöhten Prüfungsaufwand höhere Prüfungshonorare fordern. ${ }^{1642}$ Gleichzeitig können sich abschlusspolitische Ergebnisspaltungen bei steigenden Prüfungshonoraren abschwächen, da Vorstände bei höheren Prüfungshonoraren möglicherweise intensivere Prüfungen durch die Abschlussprüfer antizipieren. Folglich ist unklar, ob unerwartete bereinigte Betriebsergebnismargen oder die Höhe der Prüfungshonorare als abhängige Variable anzusehen sind.

(8) In der vorliegenden Arbeit werden etwaige Anreize für abschlusspolitische Ergebnisspaltungen separat untersucht. In der Realität beruhen die Entscheidungen der Vorstände für abschlusspolitische Ergebnisspaltungen jedoch regelmäßig auf kombinierten Anreizen. Ebenso werden die internen Corporate-Governance-Mechanismen und Qualitätsmerkmale der Abschlussprüfungen isoliert untersucht, obwohl sich deren präventive Wirkungen typischerweise gegenseitig verstärken. ${ }^{1643}$ Die Stichprobe in der vorliegenden Arbeit ist jedoch zu klein, um kombinierte Anreize zielgerichtet untersuchen zu können.

\subsection{Kritische Würdigung der Existenz von abschlusspolitischen Ergebnisspaltun- gen mit aufgegebenen Geschäftsbereichen}

In der vorliegenden Arbeit können abschlusspolitische Ergebnisspaltungen weder zum Erhöhen von Ergebnisgrößen aus den fortgeführten Geschäftsbereichen noch zum Erreichen von Referenzwerten belegt werden. ${ }^{1644}$ Ähnlich können die australischen Studien von Seve (2016) und Chagnaadorj (2018) mit ihren ursprünglichen Stichproben abschlusspolitische Ergebnisspaltungen unter dem IFRS 5 weder zum Erhöhen von Ergebnisgrö-

1642 Im Abschnitt 11.2 wird aber zumindest zwischen der Existenz von aufgegebenen Geschäftsbereichen und der Höhe der Prüfungshonorare kein Zusammenhang festgestellt.

1643 Allerdings weist Denis, D. K. (2001), S. 198/208/210 f. darauf hin, dass keine Kombination von Corporate-Governance-Mechanismen existiert, die für alle Unternehmen optimal ist.

1644 Abschlusspolitische Ergebnisspaltungen beim Erhöhen der Ergebnisse aus den fortgeführten Geschäftsbereichen können im Abschnitt 11.2 nicht einmal bei fehlenden oder ungeeigneten CorporateGovernance-Mechanismen und Qualitätsmerkmalen der Abschlussprüfungen festgestellt werden. 
ßen aus den fortgeführten Geschäftsbereichen noch zum Erreichen von Referenzwerten nachweisen. Diese Nachweise gelingen in den australischen Studien nur, indem die ursprünglichen Stichproben durch eine Vergleichsgruppenanalyse respektive durch den Ausschluss von Beobachtungen, die keine aufgegebenen Geschäftsbereiche enthalten, verkleinert werden. ${ }^{1645}$ Eine Vergleichsgruppenanalyse oder der Ausschluss von Beobachtungen, die keine aufgegebenen Geschäftsbereiche ausweisen, sind in der vorliegenden Arbeit aufgrund der kleinen Stichprobe jedoch nicht praktikabel. Demgegenüber können die USamerikanischen Studien abschlusspolitische Ergebnisspaltungen sowohl zum Erhöhen von Ergebnisgrößen aus den fortgeführten Geschäftsbereichen als auch zum Erreichen von Referenzwerten unter der APB Opinion No. 30, die dem IFRS 5 ähnelte, relativ leicht nachweisen. ${ }^{1646}$ Für den leichteren Nachweis von abschlusspolitischen Ergebnisspaltungen unter der APB Opinion No. 30 kann es mehrere Gründe geben: ${ }^{1647}$

(1) Die Stichprobe in der vorliegenden Arbeit umfasst ausschließlich Technologieunternehmen, die zwischen dem Jahr 2007 und 2014 im CDAX notiert waren. Dagegen werden in den Studien zur APB Opinion No. 30 mehrere Branchen und längere Zeiträume untersucht, wodurch die Feststellung von abschlusspolitischen Ergebnisspaltungen wahrscheinlicher wird.

(2) Die Stichprobe in der vorliegenden Arbeit enthält lediglich 325 Beobachtungen, wohingegen die Studien zur APB Opinion No. 30 typischerweise tausende Beobachtungen auswerten. ${ }^{1648}$ Aus statistischer Sicht werden mit zunehmendem Stichprobenumfang signifikante Ergebnisse c. p. wahrscheinlicher, ${ }^{1649}$ da die Teststatistiken im Zähler die Regressionskoeffzienten und im Nenner deren Standardfehler enthalten. Diese Standardfehler sinken mit zunehmendem Stichprobenumfang c. p., ${ }^{1650}$ wodurch die Teststatistiken und die Signifikanzen steigen. ${ }^{1651}$ Folglich ist die Feststellung von abschlusspolitischen Ergebnisspaltungen in der vorliegenden Arbeit mit der relativ kleinen Stichprobe tendenziell unwahrscheinlicher als in vergleichbaren Studien mit deutlich größeren Stichproben. Dem Argument, dass signifikante Ergebnisse in kleinen Stichproben c. p. seltener als in großen Stichproben auftreten, kann jedoch entgegnet werden, dass die Datenqualität aufgrund der manuellen Datenerhebung und zahlreichen Plausibilitätskontrollen in der vorliegenden Studie deutlich höher als in den vergleichbaren Studien sein dürfte. ${ }^{1652}$ Somit erschwert die kleine Stichprobe einerseits

1645 Siehe zum Vorherigen den Abschnitt 2.5.

1646 Siehe den Abschnitt 2.3.

1647 Allgemein wissenschaftstheoretisch hierzu Hager, W. (1992), S. 12 f.

1648 Siehe die Tab. 2.

1649 Vgl. Amrhein, V./Korner-Nievergelt, F./Roth, T. (2017), S. 13/21; Bortz, J./Döring, N. (2006), S. 27 f.; Gill, J. (1999), S. 657 f.; Lüken, J./Schimmelpfennig, H. (2017), o. S. Vorsichtiger Macdonald, R. R. (1997), S. 339/341. Kritisch Oakes, M. (1986), S. 22-30.

1650 Siehe Wooldridge, J. M. (2020), S. 95/132 f.

1651 Vgl. Bortz, J./Döring, N. (2006), S. 603.

1652 Siehe zur Datenqualität auch den Abschnitt 11.3. 
die Ermittlung von signifikanten Zusammenhängen, andererseits senkt die höhere Datenqualität das Risiko von Fehlschlüssen. Im Gegensatz dazu beziehen vergleichbare Studien ihre Werte typischerweise aus Datenbanken ${ }^{1653}$ und nehmen teilweise ein Winsorizing vor, mit dem Werte unter- und oberhalb bestimmter Quantile auf die jeweiligen Quantilwerte festgelegt werden. ${ }^{1654}$

(3) Abschlusspolitische Ergebnisspaltungen zum Erhöhen der Ergebnisse aus den fortgeführten Geschäftsbereichen oder zum Erreichen von Referenzwerten gelten in der vorliegenden Arbeit nur dann als belegt, wenn negative Ergebnismargen aus den aufgegebenen Geschäftsbereichen (a) unerwartet hohe bereinigte Betriebsergebnismargen im Geschäftsjahr der Aufgaben der Geschäftsbereiche und (b) ein unerwartetes Absinken der bereinigten Betriebsergebnismargen in den Geschäftsjahren nach dem Abgang der Geschäftsbereiche erklären können. Inhaltlich wird (a) als notwendige und (b) als hinreichende Bedingung bezeichnet. In der vorliegenden Arbeit sind die notwendigen Bedingungen für abschlusspolitische Ergebnisspaltungen zum Erhöhen der Ergebnisse aus den fortgeführten Geschäftsbereichen und zum Erreichen von Referenzwerten erfüllt. Da die zugehörigen hinreichenden Bedingungen jedoch nicht erfüllt sind, gilt der Nachweis von abschlusspolitischen Ergebnisspaltungen zum Erhöhen der Ergebnisse aus den fortgeführten Geschäftsbereichen oder zum Erreichen von Referenzwerten als gescheitert. Dagegen scheint es Ji/Potepa/Rozenbaum (2019) auszureichen, wenn eine der beiden Bedingungen erfüllt ist. ${ }^{1655}$

(4) In der vorliegenden Arbeit wird streng darauf geachtet, dass die bereinigten Betriebsergebnismargen, die sich per Definition nur auf die fortgeführten Geschäftsbereiche beziehen, ausschließlich durch unabhängige Variablen geschätzt werden, die ebenfalls aus den fortgeführten Geschäftsbereichen stammen. ${ }^{1656}$ Diese scharfen Abgrenzungen der Variablen aus den aufgegebenen und fortgeführten Geschäftsbereiche gewährleistet, dass für Beobachtungen mit aufgegebenen Geschäftsbereichen die erwarteten bereinigten Betriebsergebnismargen und darauf aufbauend die unerwarteten bereinigten Betriebsergebnismargen nicht systematisch anders berechnet werden als für Beobachtungen ohne aufgegebene Geschäftsbereiche. Dagegen scheinen die Variablen aus den aufgegebenen und fortgeführten Geschäftsbereichen in vergleichbaren Studi-

1653 Siehe Barua, A./Lin, S./Sbaraglia, A. M. (2010), S. 1492; Chagnaadorj, O. (2018), S. 24; Curtis, A./McVay, S./Wolfe, M. (2014), S. 195; Darrough, M./Lee, Y. G./Oh, H. I. (2019), S. 189; Ji, Y./Potepa, J./Rozenbaum, O. (2019), S. 8 f.; Rapaccioli, D./Schiff, A. (1991), S. 54; Seve, F. (2016), S. 124; Skousen, C./Sun, L./Wu, K. (2019), S. 118. Eine Ausnahme bildet die Studie von Anthonius/Murwaningsari (2018), S. 111, auf die im Abschnitt 2.4 eingegangen wird.

1654 Wie im Abschnitt 11.1 ausgeführt, bleiben die Ergebnisse in der vorliegenden Arbeit durch ein Winsorizing inhaltlich unverändert.

1655 Siehe $J i$, Y./Potepa, J./Rozenbaum, O. (2019), S. 16 f., welche die Untersuchung von abschlusspolitischen Ergebnisspaltungen beim Erreichen von nichtnegativen Ergebnissen aus den fortgeführten Geschäftsbereichen und beim Erreichen der Vorjahresergebnisse aus den fortgeführten Geschäftsbereichen fortsetzen, obwohl die notwendige Bedingung jeweils nicht erfüllt ist.

1656 Siehe den Abschnitt 8.5. 
en weniger scharf abgegrenzt zu sein, ${ }^{1657}$ wodurch die Ergebnisse verzerrt sein können. Beispielsweise können unerwartete bereinigte Betriebsergebnismargen, die mangels scharfer Abgrenzungen für Beobachtungen mit aufgegebenen Geschäftsbereichen systematisch zu hoch geschätzt werden, irrtümlich als abschlusspolitische Ergebnisspaltungen interpretiert werden.

(5) Die Stichprobe in der vorliegenden Arbeit umfasst ausschließlich Jahres- bzw. Konzernabschlüsse, die auf Grundlage der IFRS aufgestellt wurden. Dagegen werden in den US-amerikanischen Studien Abschlüsse nach US-GAAP untersucht. Obwohl sich die Rechnungslegungsnormen für aufgegebene Geschäftsbereiche nach IFRS und USGAAP teilweise ähneln, ${ }^{1658}$ können sie in der Praxis unterschiedlich ausgelegt werden. ${ }^{1659}$ Konkret können die Ermessensspielräume in den USA weiter als in Deutschland ausgelegt werden, wodurch sich abschlusspolitische Ergebnisspaltungen in den USA leichter nachweisen lassen würden. ${ }^{1660}$

(6) Während sich die vorliegende Studie auf den deutschen Rechtsraum bezieht, untersuchen die vergleichbaren Studien den US-amerikanischen Rechtsraum. Die institutionellen Rahmenbedingungen in den USA können abschlusspolitische Ergebnisspaltungen begünstigen, wohingegen die institutionellen Rahmenbedingungen in Deutschland abschlusspolitischen Ergebnisspaltungen abträglich sein können. Da der US-amerikanische Aktienmarkt im Allgemeinen höher entwickelt ist, können Eigenkapitalgeber dort mehr Einfluss als in Deutschland haben, wo Fremdkapitalgeber traditionell eine wichtige Rolle spielen. Zudem können Analystenvorhersagen in den USA besser als in Deutschland verfügbar sein und somit wichtigere Referenzwerte bilden, ${ }^{1661}$ wodurch der US-amerikanische Aktienmarkt mehr Druck auf Unternehmensleitungen ausüben und diese möglicherweise $\mathrm{zu}$ abschlusspolitischen Ergebnisspaltungen verleiten kann. Gegenläufig dazu dürften insbesondere die externen Corporate-Governance-Mechanismen in den USA wirksamer als in Deutschland sein. Beispielsweise könnten die Märkte für Unternehmensleitung, welche die Unternehmensleitungen disziplinieren können, ${ }^{1662}$ in den USA wettbewerbsintensiver als in Deutschland sein. Zudem werden den USA tendenziell strengere Haftungsregeln und eine höhere Durchsetzungsstärke von Gesetzen als Deutschland zugeschrieben, wodurch aufgedeckte und sanktionierte abschlusspolitische Ergebnisspaltungen wahr-

1657 Vgl. Barua, A./Lin, S./Sbaraglia, A. M. (2010), S. 1492; Chagnaadorj, O. (2018), S. 48 f.; Darrough, M./Lee, Y. G./Oh, H. I. (2019), S. 190/205 f.; Ji, Y./Potepa, J./Rozenbaum, O. (2019), S. 12/29 f.; Seve, F. (2016), S. 90-93; Skousen, C./Sun, L./Wu, K. (2019), S. 117 f. Ausnahmen bilden Curtis, A./McVay, S./Wolfe, M. (2014), S. 197 f.; Rapaccioli, D./Schiff, A. (1991), S. 54.

1658 Siehe den Abschnitt 2.2.

1659 Vgl. Zülch, H./Lienau, A. (2004), S. 449.

1660 Allgemeiner hierzu Ruhnke, K./Simons, D. (2018), S. 368.

$1661 \mathrm{Im}$ Abschnitt 9.3 wurde darauf hingewiesen, dass für zahlreiche Beobachtungen in der vorliegenden Arbeit keine Analystenvorhersagen vorhanden sind.

1662 Externe Corporate-Governance-Mechanismen werden im Unterabschnitt 3.2.3 ausführlich diskutiert. 
scheinlicher werden. Folglich können abschlusspolitische Ergebnisspaltungen in den USA kostenintensiver und somit unattraktiver als in Deutschland sein. Insgesamt widersprechen diese rechtlichen Rahmenbedingungen und die wettbewerbsintensiveren Märkte für Unternehmensleitung etwaigen institutionellen Rahmenbedingungen in den USA, die abschlusspolitische Ergebnisspaltungen im Vergleich zu Deutschland begünstigen.

(7) Der deutsche Arbeitnehmerschutz kann Verkäufe von aufgegebenen Geschäftsbereichen gegenüber Stilllegungen begünstigen, da Verkäufe typischerweise mit weniger umfangreichen Entlassungen als Stilllegungen verbunden sind, für die in Deutschland im globalen Vergleich hohe Hürden gelten. Folglich wären in Deutschland tendenziell mehr Verkaufsversuche von aufgegebenen Geschäftsbereichen als in anderen Ländern zu erwarten. Um diese Verkaufsversuche zu erleichtern, könnten Vorstände von deutschen Unternehmen häufiger als ihre Kollegen von ausländischen Unternehmen auf abschlusspolitische Ergebnisspaltungen verzichten. Schließlich lassen abschlusspolitische Ergebnisspaltungen zum Erhöhen der Ergebnisse aus den fortgeführten Geschäftsbereichen und zum Erreichen von Referenzwerten die aufgegebenen Geschäftsbereiche für potenzielle Käufer unnötig unattraktiv erscheinen. ${ }^{1663}$

(8) In Deutschland könnten abschlusspolitische Ergebnisspaltungen weniger im Bewusstsein als im Ausland sein. In diesem Fall ließen sich abschlusspolitische Ergebnisspaltungen mit aufgegebenen Geschäftsbereichen nicht messen, da sie unter deutschen Vorständen wenig bekannt wären und somit kaum zur Anwendung kommen würden. Die geringe Bekanntheit von abschlusspolitischen Ergebnisspaltungen mit aufgegebenen Geschäftsbereichen im deutschen Rechtsraum könnte dadurch begünstigt werden, dass abschlusspolitische Ergebnisspaltungen im Vergleich zu anderen abschlusspolitischen Maßnahmen erst seit wenigen Jahren und zunächst nur im Ausland untersucht wurden. ${ }^{1664}$ Allerdings gibt es mehrere Gründe, warum eine fehlende Bekanntheit von abschlusspolitischen Ergebnisspaltungen in Deutschland nicht deren fehlende Feststellung erklären kann. Erstens wurden in der vorliegenden Arbeit abschlusspolitische Ergebnisspaltungen bei ineffizienten Größen der fortgeführten Geschäftsbereiche festgestellt. Diese Feststellung widerspricht einer generellen Unkenntnis von abschlusspolitischen Ergebnisspaltungen im deutschen Rechtsraum. Zweitens sollten etwaige Vorteile von abschlusspolitischen Ergebnisspaltungen von Vorständen spätestens bei den Aufgaben von Geschäftsbereichen auch ohne Vorkenntnisse erkennbar sein, da abschlusspolitische Ergebnisspaltungen leicht zu praktizieren sind. Drittens sollten die ausgeprägten wirtschaftlichen Verflechtungen zwischen Deutschland und den USA die Bekanntheit von abschlusspolitischen Ergebnisspaltungen zügig angleichen.

1663 Ähnlich Keitz, I. v./Heyd, R. (2017), IFRS 5, Tz. 105. Ganz allgemein Pfleger, G. (1991), S. 25.

1664 Analog zum Einfluss der empirischen Forschung im Prüfungswesen auf menschliche Verhaltensweisen Ruhnke, K. (2000), S. 256, Fn. 141. 
Die oben genannten Gründe, warum in der vorliegenden Arbeit abschlusspolitische Ergebnisspaltungen sowohl zum Erhöhen der Ergebnisse aus den fortgeführten Geschäftsbereichen als auch zum Erreichen von Referenzwerten nicht festgestellt werden können, lassen sich in zwei grobe Kategorien aufteilen. Die Gründe (1) bis (3) zielen darauf ab, dass entsprechende abschlusspolitische Ergebnisspaltungen aufgrund unzureichender Messinstrumente nicht belegt werden können, obwohl sie möglicherweise existieren. ${ }^{1665}$ Dagegen wird mit den Gründen (4) bis (8) erläutert, warum abschlusspolitische Ergebnisspaltungen sowohl zum Erhöhen der Ergebnisse aus den fortgeführten Geschäftsbereichen als auch zum Erreichen von Referenzwerten in Deutschland evtl. nicht existieren. Zusammengefasst können in der vorliegenden Arbeit abschlusspolitische Ergebnisspaltungen zum Erhöhen der Ergebnisse aus den fortgeführten Geschäftsbereichen und zum Erreichen von Referenzwerten nicht nachgewiesen werden, da sie entweder nicht gemessen werden können oder nicht existieren.

Interessanterweise können abschlusspolitische Ergebnisspaltungen jedoch bei einer alternativ gemessenen abhängigen Variable für die hinreichende Bedingung sowohl beim Erhöhen der Ergebnisse aus den fortgeführten Geschäftsbereichen als auch beim Erreichen von Referenzwerten festgestellt werden. ${ }^{1666}$ Diese alternative abhängige Variable für die hinreichende Bedingung, die auf der abhängigen Variable für die notwendige Bedingung aufbaut, lässt sich inhaltlich intuitiver und ökonometrisch einfacher messen als die entsprechende abhängige Variable, die außerhalb des Robustheitstests (3) in enger Anlehnung an vergleichbare Studien verwendet wird. Allerdings wird bei dieser alternativ gemessenen abhängigen Variable aus zwei Gründen zur Vorsicht geraten. Erstens ist die alternative Messung derart naheliegend, dass sich die Frage aufdrängt, warum sie in vergleichbaren Studien nicht verwendet wurde. Eine mögliche Antwort lautet, dass die alternative Messung mit logischen oder ökonometrischen Problemen verbunden ist, die der Verfasser der vorliegenden Arbeit übersieht. Zweitens versagen bei der alternativen Messung nahezu sämtliche Präventivmaßnahmen für abschlusspolitische Ergebnisspaltungen zum Erhöhen der Ergebnisse aus den fortgeführten Geschäftsbereichen und zum Erreichen von Referenzwerten. Dieses Versagen der Präventivmaßnahmen ist so umfangreich, ${ }^{1667}$ dass es unplausibel erscheint und die befürchteten logischen oder ökonometrischen Probleme tendenziell bekräftigt.

Abseits von Robustheitstests kann die Existenz von abschlusspolitischen Ergebnisspaltungen mit aufgegebenen Geschäftsbereichen in der vorliegenden Arbeit nur unter der Bedingung von ineffizienten Größen der fortgeführten Geschäftsbereiche festgestellt werden. Aus Sicht der Prinzipal-Agenten-Theorie können ineffiziente Unternehmensgrößen die Eigennutzen der Vorstände erhöhen und somit Motive für abschlusspolitische Ergebnisspal-

1665 Allgemein hierzu Bortz, J./Döring, N. (2006), S. 27; Erdfelder, E./Bredenkamp, J. (1994), S. 643 f. 1666 Siehe ausführlich den Abschnitt 11.1.

1667 Vgl. die Tab. 42. 
tungen bilden. Unternehmensgrößen unterhalb der effizienten Maße kann die Eigennutzen der Vorstände erhöhen, da sich kleinere Unternehmen certeris paribus leichter führen lassen und die Vorstände dadurch weniger Arbeitsleid und mehr Freizeit genießen können. Unternehmensgrößen oberhalb der effizienten Maße können die Eigennutzen der Vorstände erhöhen, da mit den Unternehmensgrößen typischerweise die Vergütungen der Vorstände, ihr soziales Prestige sowie der Umfang von ihren Statussymbolen steigt. ${ }^{1668}$ Vorstände können sowohl ineffizient kleine als auch ineffizient große Unternehmensgrößen zumindest kurzfristig verschleiern, indem sie Aufwendungen aus den fortgeführten Geschäftsbereichen abschlusspolitisch den aufgegebenen Geschäftsbereichen zuordnen. Dadurch erscheinen die fortgeführten Geschäftsbereiche als profitabler als sie tatsächlich sind und die ineffizienten Unternehmensgrößen sind für Außenstehende schwerer zu erkennen. ${ }^{1669}$ Empirisch wird die Ineffizienz der Unternehmensgrößen in der vorliegenden Arbeit zunächst mit der Interaktionsvariable $B M{ }_{-} 1_{i, t}$ operationalisiert. $B M_{-} G 1_{i, t}$ wird der Wert 1 zugewiesen, falls der Buch- den Marktwert des Eigenkapitals übersteigt. Andernfalls wird $B M_{-} G 1_{i, t}$ mit dem Wert 0 kodiert. Die Idee hinter dieser Operationalisierung lautet, dass die Kapitalmärkte bei $B M_{-} G 1_{i, t}=1$ negative Gewinnerwartungen für die Unternehmen zu haben scheinen, die typischerweise aus ineffizienten Unternehmensgrößen resultieren. ${ }^{1670}$ Mit dieser Operationalisierung von ineffizienten Unternehmensgrößen konnten abschlusspolitische Ergebnisspaltungen festgestellt werden.

Die obige Argumentation, dass Vorstände gemäß der Prinzipal-Agenten-Theorie ineffiziente Unternehmensgrößen beibehalten wollen und mit abschlusspolitischen Ergebnisspaltungen verschleiern können, mag plausibel wirken und kann empirisch untermauert werden. Allerdings wirken auch die Argumentationen für abschlusspolitische Ergebnisspaltungen zum Erhöhen der Ergebnisse aus den fortgeführten Geschäftsbereichen und zum Erreichen von Referenzwerten plausibel, können in den vorliegenden Arbeit aber empirisch nicht belegt werden. Vor diesem Hintergrund kann die Frage gestellt werden, warum in der vorliegenden Arbeit abschlusspolitische Ergebnisspaltungen ausgerechnet bei ineffizienten Unternehmensgrößen festgestellt werden. Eine mögliche Antwort lautet, dass ineffiziente Unternehmensgrößen mit der Interaktionsvariable $B M_{-} G 1_{i, t}$ zwar einfach, aber zu indirekt operationalisiert sind. So müssen negative Gewinnerwartungen der Kapitalmärkte, welche die Marktwerte des Eigenkapitals von Unternehmen unter den entsprechenden Buchwert senken können, nicht zwingend aus ineffizienten Unternehmensgrößen resultieren. Vielmehr können nicht vollständig rationale Kapitalmärkte bspw. aufgrund von Stimmungen und Gerüchten negative Gewinnerwartungen für Unternehmen entwickeln, obwohl deren Größen i. S. d. Transaktionskostentheorie effizient sind. Um das Problem eines möglicherweise zu indirekten Ineffizienzmaßes für die Unternehmensgrö-

1668 Siehe zum Vorherigen den Unterabschnitt 5.2.3.

1669 Siehe zum Vorherigen den Unterabschnitt 5.2.8.

1670 Der Zusammenhang zwischen negativen Gewinnerwartungen und ineffizienten Unternehmensgrößen wird im Abschnitt 9.2 ausführlich beschrieben. 
ßen einzudämmen, wird die Ineffizienz der Unternehmensgrößen im Robustheitstest (4) anhand des Survivor-Principle direkter ermittelt. ${ }^{1671}$ Hierfür wird der Interaktionsvariable $G R_{-} I E F F_{F, i, t}$ der Wert 1 zugewiesen, falls eine Beobachtung einer Größenklasse angehört, die im Zeitverlauf an Markanteilen und Mitgliedern verliert. Andernfalls wird $G R \_I E F F_{F, i, t}$ mit dem Wert 0 kodiert. Hinter dieser Operationalisierung steht die Idee, dass die brancheninternen Marktanteile der Unternehmen mit den ineffizientesten Größen im Zeitverlauf am stärksten sinken, während die Marktanteile der Konkurrenten mit effizienten Größen steigen. ${ }^{1672}$ Da die Testergebnisse mit den Interaktionsvariablen $B M_{-} G 1_{i, t}$ und $G R_{-} I E F F_{F, i, t}$ inhaltlich identisch sind, scheinen ineffiziente Unternehmensgrößen abschlusspolitische Ergebnisspaltungen tatsächlich zu begünstigen.

Dem Verfasser der vorliegenden Arbeit ist keine andere Studie bekannt, die abschlusspolitische Ergebnisspaltungen bei ineffizienten Unternehmensgrößen untersucht. Es wäre interessant zu wissen, ob sich abschlusspolitische Ergebnisspaltungen bei ineffizienten Unternehmensgrößen in den Stichproben von bisherigen und zukünftigen Studien replizieren lassen. Im Gegensatz zur vorliegenden Arbeit können vergleichbare Studien typischerweise abschlusspolitische Ergebnisspaltungen beim Erhöhen der Ergebnisses aus den fortgeführten Geschäftsbereichen und beim Erreichen von Referenzwerten nachweisen. ${ }^{1673}$ Da die Unternehmen in diesen Stichproben stärker zu abschlusspolitischen Ergebnisspaltungen als in der vorliegenden Stichprobe neigen, könnte in diesen Stichproben aus vergleichbaren Studien möglicherweise ebenfalls abschlusspolitische Ergebnisspaltungen bei ineffizienten Unternehmensgrößen nachgewiesen werden.

Im Gegensatz zu vergleichbaren Studien werden in der vorliegenden Arbeit zudem Cookie Jars und Big Baths als Motive für abschlusspolitische Ergebnisspaltungen untersucht. Abweichend von den bisherigen Motiven ordnen Vorstände bei Cookie Jars und Big Baths Aufwendungen aus den aufgegebenen Geschäftsbereichen abschlusspolitisch den fortgeführten Geschäftsbereichen zu, um Ertragsreserven für darauffolgende Geschäftsjahre aufzubauen und die Erwartungshaltung der Kapitalmärkte für die zukünftige Ertragslage zu begrenzen. Dabei sinken die unerwarteten bereinigten Betriebsergebnismargen, während die Ergebnismargen aus den aufgegebenen Geschäftsbereichen tendenziell positiv werden. Der Unterschied zwischen Cookie Jars und Big Baths ist, dass Vorstände bei Cookies Jar bestimmte Referenzwerte für Ertragsgrößen nicht zu weit übertreffen wollen, wohingegen sie bei Big Baths diese Referenzwerte absichtlich verfehlen. Empirisch können abschluss-

1671 Allerdings bezeichnen Danos, P./Eichenseher, J. W. (1982), S. 605 auch das Survivor Principle als indirekte Messmethode für effiziente Unternehmensgrößen, da es etwaige Kostenvorteile anhand von Marktanteilen approximiert. Zudem betrachtet Weiss, L. W. (1964), S. 247 das Survivor Principle für die Identifizierung von effizienten Größen von Fabrikanlagen als besser geeignet als für die Identifizierung von effizienten Unternehmensgrößen, da mehrere Fabrikanlagen in einzelnen Unternehmen gebündelt sein können und deren möglicherweise unterschiedliche Effizienzklassen sich vermischen können.

1672 Kritisch merkt Weiss, L. W. (1964), S. 246 an, dass Unternehmen trotz ineffizienten Größen überleben können, indem sie bspw. ihre Zulieferer ausbeuten.

1673 Siehe den Abschnitt 2.6. 
politische Ergebnisspaltungen jedoch weder bei Cookie Jars noch bei Big Baths nachgewiesen werden. Wie bereits bei abschlusspolitischen Ergebnisspaltungen zum Erhöhen der Ergebnisse aus den fortgeführten Geschäftsbereichen und zum Erreichen von Referenzwerten, kann auch der fehlende Nachweis von abschlusspolitischen Ergebnisspaltungen zwecks Cookie Jars und Big Baths zwei übergeordnete Ursachen haben: Entweder können abschlusspolitische Ergebnisspaltungen bei Cookie Jars und Big Baths nicht gemessen werden oder sie existieren nicht. Die empirische Messung kann vor allem aufgrund der ausschließlichen Untersuchung von Technologieunternehmen und der kleinen Stichprobe scheitern. ${ }^{1674}$ Dagegen können bei Cookie Jars und Big Baths abschlusspolitische Ergebnisspaltungen nicht existieren, da deren Eignung zum Erreichen dieser abschlusspolitischen Ziele schwerer zu erkennen ist als bei den bisher betrachteten Zielen. Darüber hinaus lässt sich der zukünftige Entfall der Aufwendungen aus den aufgegebenen Geschäftsbereichen, die abschlusspolitisch den fortgeführten Geschäftsbereichen zugeordnet werden, zeitlich nur schwer steuern. Sobald die aufgegebenen Geschäftsbereiche abgegangen sind, werden die fortgeführten Geschäftsbereiche um die Aufwendungen aus den aufgegebenen Geschäftsbereichen, die zwischenzeitlich abschlusspolitisch den fortgeführten Geschäftsbereichen zugeordnet wurden, entlastet. Diese Zeitpunkte des Abgangs der Geschäftsbereiche durch Verkäufe oder Stilllegungen lassen sich leichter objektivieren als bspw. das Ende von Restrukturierungsphasen und Auflösungen von entsprechenden Rückstellungen, mit denen ebenfalls abschlusspolitische Ertragsreserven i. S. v. Cookie Jars oder Big Baths gebildet werden können.

Ferner stimmt der fehlende Nachweis von abschlusspolitischen Ergebnisspaltungen bei Cookie Jars mit Barua/Lin/Sbaraglia (2010) überein, die bei positiven Ergebnissen aus den aufgegebenen Geschäftsbereichen ebenfalls keine abschlusspolitischen Ergebnisspaltungen belegen können: „We believe that classification shifting can take place when firms report positive discontinued operations, although we do not find the predicted results. “1675 Da abschlusspolitische Ergebnisspaltungen zwecks Cookie Jars im Übrigen nur bei positiven Ergebnismargen aus den aufgegebenen Geschäftsbereichen als naheliegend erscheinen, ${ }^{1676}$ wird in der vorliegenden Arbeit in Anlehnung an Barua/Lin/Sbaraglia (2010) implizit ebenfalls untersucht, ob abschlusspolitische Ergebnisspaltungen zum Erreichen von

1674 Siehe den vorliegenden Abschnitt.

1675 Barua, A./Lin, S./Sbaraglia, A. M. (2010), S. 1501, Fn. 17. Auch Ji, Y./Potepa, J./Rozenbaum, O. (2019), S. 21 f. können bei positiven Ergebnissen aus den aufgegebenen Geschäftsbereichen keine abschlusspolitischen Ergebnisspaltungen nachweisen.

1676 Wenn Aufwendungen aus den aufgegebenen Geschäftsbereichen abschlusspolitisch unter den fortgeführten Geschäftsbereichen ausgewiesen werden, werden die Ergebnisse aus den aufgegebenen Geschäftsbereichen tendenziell positiv. 
Referenzwerten auch bei positiven ${ }^{1677}$ Ergebnissen aus den aufgegebenen Geschäftsbereichen auftreten. ${ }^{1678}$

In der juristisch geprägten Fachliteratur zu IFRS 5 ist strittig, ob Ergebnisspaltungen mit aufgegebenen Geschäftsbereichen überhaupt abschlusspolitisch sein können. ${ }^{1679}$ Die diesbezügliche Mindermeinung von Küting/Wirth (2006) verneint Ermessensspielräume bei Ergebnisspaltungen mit aufgegebenen Geschäftsbereichen und hält diese entweder für normenkonform oder -widrig. Demnach seien Unternehmensteile, deren Aufwendungen und Erträge sich nicht klar von den restlichen Unternehmensteilen trennen lassen, gemäß der Definition in IFRS 5.31 keine aufgegebenen Geschäftsbereiche. ${ }^{1680}$ Trotz dieses logisch schlüssigen Arguments identifiziert die herrschende Meinung bei den praktischen Anwendungen der Ergebnisspaltungen mit aufgegebenen Geschäftsbereichen Ermessensspielräume und hält eine diesbezügliche Abschlusspolitik daher für möglich. ${ }^{1681}$ Die Tatsache, dass in der vorliegenden Arbeit abschlusspolitischen Ergebnisspaltungen nur bei ineffizienten Unternehmensgrößen, nicht jedoch beim Erhöhen der Ergebnisse aus den fortgeführten Geschäftsbereichen, beim Erreichen von Referenzwerten, bei Cookie Jars und bei Big Baths belegt werden können, bekräftigt vor allem die Mindermeinung von Küting/Wirth (2006). Im Sinne der Mindermeinung können die in der vorliegenden Arbeit empirisch festgestellten Ergebnisspaltungen bei ineffizienten Unternehmensgrößen auf Verstöße gegen IFRS 5.31 hindeuten. Alleine schon aufgrund der im Abschnitt 11.3 diskutierten Grenzen der empirischen Untersuchung kann die vorliegende Arbeit jedoch keinesfalls als Beweis für unzulässige Ergebnisspaltungen bei ineffizienten Unternehmensgrößen interpretiert werden. Für einen derartigen Beweis wären umfangreiche unternehmensinterne Daten erforderlich und selbst mit diesen dürften i. S.d. herrschenden Meinung „vielfach unterschiedliche Lösungen vertreten [werden] können. “1682

Neben abschlusspolitischen Ergebnisspaltungen wird in der Fachliteratur zu IFRS 5 auffällig oft ein abschlusspolitischer Ausweis von aufgegebenen Geschäftsbereichen vermutet. ${ }^{1683}$ Dabei werden die Ermessensspielräume bei der Definition eines aufgegebenen Geschäftsbereichs so ausgelegt, dass aufgegebene Geschäftsbereiche nur dann ausgewiesen werden, wenn sie den abschlusspolitischen Zielen der Vorstände dienen. Bestehen die abschlusspolitischen Ziele bspw. im Erhöhen der Ergebnisse aus den fortgeführten Geschäftsbereichen, können Vorstände defizitäre Unternehmensteile möglichst früh als aufgegebe-

1677 Aufgrund der theoretischen Überlegungen im Abschnitt 8.3 und der empirischen Erkenntnisse im Abschnitt 9.1 werden abschlusspolitische Ergebnisspaltungen beim Erreichen von Referenzwerten in der Regressionsgleichung (19a) nur bei negativen Ergebnissen aus den aufgegebenen Geschäftsbereichen untersucht.

1678 Hierzu Barua, A./Lin, S./Sbaraglia, A. M. (2010), S. 1501, Fn. 17. Auch Darrough, M./Lee, Y. G./Oh, H. I. (2019), S. $193 \mathrm{f}$.

1679 Siehe den Unterabschnitt 4.3.2.

1680 Zum Vorhergehenden Küting, K./Wirth, J. (2006), S. 725, Fn. 62.

1681 Siehe den Unterabschnitt 4.3.2.

1682 Kessler, H./Leinen, M. (2006), S. 566. Analog Barnea, A./Ronen, J./Sadan, S. (1976), S. 111 f.

1683 Siehe den Abschnitt 11.2. 
ne Geschäftsbereiche deklarieren, wodurch die negativen Ergebnisbeiträge der defizitären Unternehmensteile aus den Ergebnissen aus den fortgeführten Geschäftsbereichen herausgefiltert werden. In der ergänzenden Analyse (2) konnte ein abschlusspolitischer Ausweis von aufgegebenen Geschäftsbereichen empirisch jedoch nicht belegt werden. Der gescheiterte Nachweis kann aus dem dichotomen Charakter des abschlusspolitischen Ausweises von aufgegebenen Geschäftsbereichen resultieren, der mit einem erheblichen Informationsverlust verbunden ist. Beispielsweise werden hinsichtlich der Existenz von aufgegebenen Geschäftsbereichen Unternehmen mit Ergebnissen aus den aufgegebenen Geschäftsbereichen in Höhe von -1 TEUR genauso behandelt wie Unternehmen mit Ergebnissen aus den aufgegebenen Geschäftsbereichen in Höhe von -1.000 TEUR. Allerdings ist es unwahrscheinlich, dass Vorstände Geschäftsbereiche abschlusspolitisch als aufgegeben deklarieren, nur um -1 TEUR aus den Ergebnissen aus den fortgeführten Geschäftsbereichen herauszufiltern. Die künstliche Nominalskalierung der Ergebnisses aus den aufgegebenen Geschäftsbereichen bei der Untersuchung eines abschlusspolitischen Ausweises von aufgegebenen Geschäftsbereichen kann gleichzeitig der Grund dafür sein, warum abschlusspolitische Ergebnisspaltungen im Gegensatz zu einem abschlusspolitischen Ausweis empirisch nachgewiesen werden können. Es ist jedoch zu beachten, dass die verhältnisskalierte Testvariable $E A G B_{i, t}$ gewissermaßen eine Doppelfunktion bezüglich dem abschlusspolitischen Ausweis von aufgegebenen Geschäftsbereichen und den abschlusspolitische Ergebnisspaltungen mit aufgegebenen Geschäftsbereichen erfüllt. Zum einen erfasst $E A G B_{i, t}$ unter der Voraussetzung des Ausweises eines aufgegebenen Geschäftsbereichs dessen Ergebnismarge und zum anderen erhält $E A G B_{i, t}$ ohne einen aufgegebenen Geschäftsbereich und somit ohne Ergebnis aus den aufgegebenen Geschäftsbereichen den Wert $0 .{ }^{1684}$ Anders ausgedrückt können abschlusspolitische Ergebnisspaltungen nur unter der Voraussetzung des Ausweises eines aufgegebenen Geschäftsbereichs existieren, der ebenfalls abschlusspolitisch sein kann. Die empirische Evidenz in der vorliegenden Arbeit widerspricht jedoch einem abschlusspolitischen Ausweis von aufgegebenen Geschäftsbereichen.

\subsection{Kritische Würdigung der Prävention von abschlusspolitischen Ergebnisspal- tungen mit aufgegebenen Geschäftsbereichen}

Nachdem die empirischen Befunde zur Existenz von abschlusspolitischen Ergebnisspaltungen diskutiert wurden, werden nun die empirischen Erkenntnisse zur Prävention von abschlusspolitischen Ergebnisspaltungen kritisch gewürdigt. Da abschlusspolitische Ergebnisspaltungen in der vorliegenden Arbeit - abgesehen vom Robustheitstest (3) - nur bei ineffizienten Unternehmensgrößen festgestellt werden, kann die Prävention von abschlusspolitischen Ergebnisspaltungen empirisch ebenfalls nur unter der Bedingung von ineffizienten Unternehmensgrößen untersucht werden. Genauer formuliert sind die empi-

1684 Analog erfassen die Testvariablen $E A G B \_N_{i, t}$ und $E A G B \_P_{i, t}$ die Höhe des negativen respektive positiven Ergebnisses aus den aufgegebenen Geschäftsbereichen und erhalten ansonsten den Wert 0. 
rischen Erkenntnisse zur Prävention von abschlusspolitischen Ergebnisspaltungen in der vorliegenden Arbeit auf diejenigen börsennotierten Technologieunternehmen im Zeitraum von 2008 bis 2013 beschränkt, die bei ineffizienten Größen der fortgeführten Geschäftsbereiche abschlusspolitische Ergebnisspaltungen mit aufgegebenen Geschäftsbereichen vornehmen. Aufgrund dieser engen Grenzen werden die empirischen Erkenntnisse zur Prävention von abschlusspolitischen Ergebnisspaltungen nachfolgend weniger intensiv als deren Existenz diskutiert.

Bezüglich der Prävention von abschlusspolitischen Ergebnisspaltungen bei ineffizienten Unternehmensgrößen überrascht zunächst, dass Aufsichtsräte i. S. v. $\mathrm{H}_{2.1}$, Eigentümerstrukturen i. S. v. $\mathrm{H}_{2.3}$ und Prüfungshonorare i. S. v. $\mathrm{H}_{2.5}$ als Präventivmaßnahmen versagen. ${ }^{1685}$ Analog zur Existenz von abschlusspolitischen Ergebnisspaltungen kann der fehlende Beleg der Prävention von abschlusspolitischen Ergebnisspaltungen bei ineffizienten Unternehmensgrößen entweder durch ungeeignete Messmethoden oder durch tatsächlich unzureichende Präventivwirkungen verursacht sein. Die Messmethoden können ungeeignet sein, da die Präventivmaßnahmen in der vorliegenden Arbeit nur mit jeweils einer Interaktionsvariable abgebildet werden, wohingegen die Motive für abschlusspolitische Ergebnisspaltungen typischerweise mit mehreren Interaktionsvariablen operationalisiert werden. ${ }^{1686}$ Tatsächlich vorhandene Präventivwirkungen können mithin übersehen werden, wenn das entsprechende Surrogat ungünstig gewählt ist. Hinsichtlich tatsächlich unzureichenden Präventivwirkungen fällt auf, dass Aufsichtsräte i. S. v. $\mathrm{H}_{2.1}$, Eigentümerstrukturen i. S. v. $\mathrm{H}_{2.3}$ und Prüfungshonorare i. S. v. $\mathrm{H}_{2.5}$, die übereinstimmend durch nominalskalierte Interaktionsvariablen abgebildet werden, als Präventivmaßnahmen scheitern. Nachfolgend wird inhaltlich näher beleuchtet, warum Aufsichtsräte i. S. v. $\mathrm{H}_{2.1}$, Eigentümerstrukturen i. S. v. $\mathrm{H}_{2.3}$ und Prüfungshonorare i. S. v. $\mathrm{H}_{2.5}$ als Präventivmaßnahmen versagen können.

Aufsichtsräte i. S. v. $\mathrm{H}_{2.1}$ verfügen über ausschließlich fixe Vergütungssysteme und über Prüfungsausschüsse mit Finanzexperten als Vorsitzende. Derartige Aufsichtsräte können abschlusspolitische Ergebnisspaltungen bei ineffizienten Unternehmensgrößen möglicherweise nicht verhindern, wenn sie im Rahmen der Aufgabe von Geschäftsbereichen zu stark mit strategischen und juristischen Themen beschäftigt sind, um sich Ergebnisspaltungen widmen zu können. Zudem können Aufsichtsräte i. S. v. $\mathrm{H}_{2.1}$ an der Prävention von abschlusspolitischen Ergebnisspaltungen scheitern, wenn sie zu selten mit aufgegebenen Geschäftsbereichen konfrontiert sind, um abschlusspolitische Ergebnisspaltungen erkennen zu können. Selbst Finanzexperten in den Prüfungsausschüssen müssen keine Experten für die Rechnungslegung zu aufgegebenen Geschäftsbereichen sein. Bezüglich der Fach-

1685 Die Alternativhypothesen $\mathrm{H}_{2.1}, \mathrm{H}_{2.3}$ und $\mathrm{H}_{2.5}$ sind in der Tab. 36 zusammengetragen.

1686 Vgl. die letzte Spalte der Tab. 28 mit der letzten Spalte der Tab. 36. Beispielsweise wird die Alternativhypothese $\mathrm{H}_{1.3}$ für die Existenz von abschlusspolitischen Ergebnisspaltungen beim Erreichen von Referenzwerten mit sechs alternativen Interaktionsvariablen abgebildet. 
kompetenz von Prüfungsausschüssen kann eine lose Analogie aus der US-amerikanischen Studie von Skousen/Sun/Wu (2019) abgeleitet werden. Unter der APB Opinion No. 30, die dem IFRS 5 ähnelte, zeigen Skousen/Sun/Wu (2019), dass sich abschlusspolitische Ergebnisspaltungen mit steigenden Fähigkeiten der Vorstände abschwächten ${ }^{1687}$ Analog könnten sich abschlusspolitische Ergebnisspaltungen mit steigenden Fähigkeiten der Prüfungsausschüsse abschwächen.

Eigentümerstrukturen i. S. v. $\mathrm{H}_{2.3}$ zeichnen sich durch mindestens einen Großaktionär aus. Möglicherweise wollen Großaktionäre abschlusspolitische Ergebnisspaltungen zum einen nicht verhindern, weil sie über direkte Informationszugänge zu Vorständen verfügen und die Rechnungslegung für die Großaktionäre somit nur von untergeordneter Bedeutung ist. Zum anderen können sich Großaktionäre, die zusätzlich über für sie strategisch oder finanziell relevantere Aktienpakete von anderen Unternehmen verfügen, ähnlich passiv wie die meisten Kleinaktionäre verhalten.

Prüfungshonorare i. S. v. $\mathrm{H}_{2.5}$ haben eine negative abnormale Höhe und fallen in Geschäftsjahren außerhalb von externen Prüferrotationen an. In der vorliegenden Arbeit wurde ein positiver Zusammenhang zwischen negativen abnormalen Prüfungshonoraren und der Unabhängigkeit der Abschlussprüfer vermutet, da Abschlussprüfer bei negativen abnormalen Prüfungshonoraren außerhalb von externen Prüferrotationen keine wirtschaftlichen Anreize zu Verlängerungen der Mandantenbeziehungen und somit zu Zugeständnissen gegenüber den Mandanten haben. Allerdings ist es ebenso denkbar, dass Abschlussprüfer auf negative abnormale Prüfungshonorare mit geringen Prüfungsanstrengungen reagieren und deshalb abschlusspolitische Ergebnisspaltungen übersehen. Unabhängig von einer abnormalen Höhe der Prüfungshonorare könnten Abschlussprüfer aufgegebene Geschäftsbereiche vernachlässigen, da sie bei deren Existenz trotz der komplexeren Abschlussprüfungen grundsätzlich keine höheren Prüfungshonorare durchsetzen können. ${ }^{1688}$

Im Gegensatz zu Aufsichtsräten i. S. v. $\mathrm{H}_{2.1}$, Eigentümerstrukturen i. S. v. $\mathrm{H}_{2.3}$ und Prüfungshonoraren i. S. v. $\mathrm{H}_{2.5}$ werden für Vorstandsvergütungen i. S. v. $\mathrm{H}_{2.2}$, Kapitalstrukturen i. S. v. $\mathrm{H}_{2.4}$ und Nichtprüfungshonoraren i. S. v. $\mathrm{H}_{2.6}$, die alle mit verhältnisskalierten Interaktionsvariablen operationalisiert werden, schwächere abschlusspolitische Ergebnisspaltungen bei ineffizienten Unternehmensgrößen festgestellt. ${ }^{1689}$ Demnach lassen sich abschlusspolitische Ergebnisspaltungen bei ineffizienten Unternehmensgrößen mit verhältnisskalierten Interaktionsvariablen leichter abschwächen als mit nominalskalierten Interaktionsvariablen verhindern. Diese Schlussfolgerung wird auch durch die ergänzende Analyse (1) gestützt, in der die verhältnisskalierten Surrogate zu nominalskalierten Surrogaten komprimiert werden, um zu testen, ob die verhältnisskalierten Surrogate bei hohen Werten,

1687 Siehe zum Vorherigen Skousen, C./Sun, L./Wu, K. (2019), S. 113-131 und den Abschnitt 2.2.

1688 Siehe die ergänzende Analyse (4).

1689 Die Alternativhypothesen $\mathrm{H}_{2.2}, \mathrm{H}_{2.4}$ und $\mathrm{H}_{2.6}$ sind in der Tab. 36 zusammengetragen. 
die eine Standardabweichung über den Mittelwerten liegen, präventiv wirken. Mit diesen hohen Werten für die verhältnisskalierten Surrogate wirken Kapitalstrukturen i. S. v. $\mathrm{H}_{2.4}$ präventiv, Vorstandsvergütungen i. S. v. $\mathrm{H}_{2.2}$ und Nichtprüfungshonorare i. S. v. $\mathrm{H}_{2.6}$ jedoch nicht. Möglicherweise können die Vorstandsvergütungen und die Nichtprüfungshonorare abschlusspolitische Ergebnisspaltungen nur in Kombination mit anderen internen Corporate-Governance-Mechanismen verhindern. ${ }^{1690}$

Die Größen der Abschlussprüfer i. S. v. $\mathrm{H}_{2.7}$ und ihre Branchenspezialisierungen i. S. v. $\mathrm{H}_{2.8}$ nehmen bei der Prävention von abschlusspolitischen Ergebnisspaltungen eine Sonderstellung ein. ${ }^{1691}$ Entgegen der herrschenden Meinung wurde mit $\mathrm{H}_{2.7}$ und $\mathrm{H}_{2.8}$ aufgrund theoretischer Argumente postuliert, dass die Größen und Branchenspezialisierungen der Abschlussprüfer etwaige abschlusspolitische Ergebnisspaltungen nicht beeinflussen. Empirisch kann $\mathrm{H}_{2.7}$ zumindest nicht widerlegt werden, da zwischen den Größen der Abschlussprüfer und abschlusspolitischen Ergebnisspaltungen bei ineffizienten Unternehmensgrößen kein Zusammenhang festgestellt werden kann. ${ }^{1692} \mathrm{H}_{2.8}$ muss dagegen abgelehnt werden, da die Branchenspezialisierungen der Abschlussprüfer abschlusspolitische Ergebnisspaltungen bei ineffizienten Unternehmensgrößen abschwächen. ${ }^{1693}$ Diese unerwarteten Abschwächungen können entweder durch ungeeignete Messmethoden irrtümlich festgestellt oder durch eine tatsächliche Wirksamkeit der Branchenspezialisierungen der Abschlussprüfer verursacht sein. Die Messmethoden können ungeeignet sein, da die Branchenspezialisierungen der Abschlussprüfer nur mit der Marktanteilsmethode gemessen werden, obwohl letztere kleine Abschlussprüfer systematisch benachteiligt. ${ }^{1694}$ Die Ausmaße dieser systematischen Benachteiligungen könnten durch die komplementäre Verwendung der Portfoliomethode abgeschätzt werden. Aufgrund von Datenrestriktionen kann die Portfoliomethode in der vorliegenden Arbeit jedoch ebenso wenig wie diejenige Methode, die auf den selbstproklamierten Branchenspezialisierungen basiert, verwendet werden. Insofern können die Branchenspezialisierungen der Abschlussprüfer in der vorliegenden Arbeit systematisch falsch gemessen sein. Allerdings können die Branchenspezialisierungen die abschlusspolitischen Ergebnisspaltungen bei ineffizienten Unternehmensgrößen tatsächlich abschwächen, falls sich die Abschlussprüfer entgegen der Prinzipal-AgentenTheorie nicht opportunistisch verhalten. ${ }^{169}$ In diesem Fall werden die besseren Fähigkeiten von branchenspezialisierten Abschlussprüfern zur Erkennung von abschlusspolitischen Ergebnisspaltungen nicht durch ihren diesbezüglich fehlenden Willen neutralisiert.

1690 Siehe hierzu den Abschnitt 11.3 und den Abschnitt 11.7.

1691 Die Alternativhypothesen $\mathrm{H}_{2.7}$ und $\mathrm{H}_{2.8}$ sind in der Tab. 36 zusammengetragen.

1692 Siehe zu den methodischen Details die Fn. 1573 und die Fn. 1574.

1693 Die ergänzende Analyse (1) zeigt jedoch, dass selbst hohe Branchenspezialisierungen der Abschlussprüfer abschlusspolitischen Ergebnisspaltungen bei ineffizienten Unternehmensgrößen nicht vollständig verhindern.

1694 Die Methoden zur Messung der Branchenspezialisierungen der Abschlussprüfer werden im Abschnitt 10.1 diskutiert.

1695 Siehe hierzu den Unterabschnitt 7.4.9. 


\subsection{Beantwortung der Forschungsfragen und Fazit}

Die vorliegende Arbeit basiert auf den zwei Forschungsfragen, ob (1) abschlusspolitische Ergebnisspaltungen mit aufgegebenen Geschäftsbereichen existieren und ob (2) sich abschlusspolitische Ergebnisspaltungen mit aufgegebenen Geschäftsbereichen verhindern lassen. ${ }^{1696}$ Beide Forschungsfragen sind zu bejahen, da (1) abschlusspolitische Ergebnisspaltungen mit aufgegebenen Geschäftsbereichen bei ineffizienten Unternehmensgrößen festgestellt werden und sich (2) diese abschlusspolitischen Ergebnisspaltungen durch hohe Verschuldungsgrade verhindern lassen. Somit können die Transaktionskostentheorie und die Prinzipal-Agenten-Theorie die Existenz und Prävention von abschlusspolitischen Ergebnisspaltungen empirisch nur ausschnittsweise erklären. ${ }^{167}$ Die Prospect Theory leistet im Hauptmodell sogar keinen empirischen Erklärungsbeitrag. ${ }^{1698}$ Der stark limitierte Aussagehalt der Theorien wird i. S. d. methodologischen Falsifikationismus jedoch nicht zum Anlass genommen, um diese Theorien zu verwerfen, sondern lediglich als Ausdruck des weiteren Forschungsbedarfs betrachtet. ${ }^{169}$

Abschließend soll auf die Relevanz der empirischen Erkenntnisse für Abschlussadressaten, Abschlussprüfer und Normengeber zurückgekommen werden. ${ }^{1700}$ Grundsätzlich können alle Rechnungslegungsnormen abschlusspolitisch eingesetzt werden. ${ }^{1701}$ Verfügen Technologieunternehmen allerdings über negatives Ergebnisse aus den aufgegebenen Geschäftsbereichen und ineffiziente Größen, sollten Abschlussadressaten und Abschlussprüfer deren Jahres- bzw. Konzernabschlüsse besonders aufmerksam analysieren, da abschlusspolitische Ergebnisspaltungen verstärkt auftreten können. ${ }^{1702}$ Entsprechende ineffiziente Unternehmensgrößen können vermutet werden, falls die Buchwerte des Eigenkapitals deren Marktwerte übersteigen. Bei zahlreichen anderen Motiven wurden in der vorliegenden Arbeit jedoch keine abschlusspolitischen Ergebnisspaltungen festgestellt. Daher sind zumindest bei Technologieunternehmen keine flächendeckenden abschlusspolitischen Ergebnisspaltungen zu erkennen. Folglich lässt sich aus der vorliegenden

1696 Siehe den Abschnitt 1.3.

1697 Für die Alternativhypothese $\mathrm{H}_{1.2}$, die empirisch zumindest vorläufig als belegt gilt, werden die ineffizienten Unternehmensgrößen aus der Transaktionskostentheorie und die Motive der Vorstände für das Verschleiern der ineffizienten Unternehmensgrößen aus der Prinzipal-Agenten-Theorie abgeleitet.

1698 Die Alternativhypothesen $\mathrm{H}_{1.3}, \mathrm{H}_{1.4}$ und $\mathrm{H}_{1.5}$, für welche die Bedeutung von Referenzwerten aus der Prospect Theory abgeleitet werden, können empirisch nicht belegt werden. Lediglich im Robustheitstest (3) werden abschlusspolitische Ergebnisspaltungen beim Erreichen von Referenzwerten vorläufig festgestellt.

1699 Vgl. Lakatos, I. (1970), S. 133 f.; Lakatos, I. (1974), S. 129-131. Auch Bortz, J./Döring, N. (2006), S. 21/27; Erdfelder, E./Bredenkamp, J. (1994), S. 643 f. Analog Köhler, A. G. (2003), S. 240. Der Bedarf an Theorien, welche die Existenz und Prävention von abschlusspolitischen Ergebnisspaltungen besser erklären können, wird auch im Abschnitt 11.7 thematisiert.

1700 Siehe den Abschnitt 1.2.

1701 Hierzu Coenenberg, A. G./Haller, A./Schultze, W. (2018), S. 1027; Küting, K. (2008), Teil H, Tz. 2102; Müller, S./Wulf, I. (2001), S. 2206; Pfleger, G. (1991), S. 25/35.

1702 Allgemeiner Almaleeh, N. M. S. (2019), S. 36. Müller, S./Wulf, I. (2001), S. 2213 vermuten bei abschlusspolitischen Maßnahmen branchenspezifische Verhaltensmuster. 
Untersuchung auch nicht ableiten, dass der IFRS 5 dringend überarbeitet werden müsse. Vielmehr könnte es ausreichen, Ergebnisspaltungen mit aufgegebenen Geschäftsbereichen weiterhin als Untersuchungsschwerpunkt der DPR bzw. deren Nachfolgerin zu verwenden. ${ }^{1703}$ Keinesfalls dürfen die empirischen Erkenntnisse in der vorliegenden Arbeit jedoch verallgemeinert werden, da sie nur für börsennotierte Technologieunternehmen gelten. Im folgenden Ausblick wird beleuchtet, wie diese und weitere Einschränkungen zukünftig überwunden werden können und welche Aspekte von aufgegebenen Geschäftsbereichen ebenfalls untersucht werden können.

\subsection{Ausblick}

Obwohl mit den Technologieunternehmen die größte Branche des CDAX im Zeitraum von 2008 bis 2013 abgedeckt wurde, verbleiben für die Stichprobe lediglich 325 Beobachtungen, von denen 42 Beobachtungen aufgegebene Geschäftsbereiche ausweisen. Die vorliegende Arbeit zeigt, dass abschlusspolitische Ergebnisspaltungen auch mit dieser kleinen Stichprobe analysiert werden können. Aufgrund der ständig wachsenden Anzahl von Jahres- bzw. Konzernabschlüssen aus neueren Geschäftsjahren lässt sich diese Analyse aber in zweierlei Hinsicht erweitern. Zum einen kann die Grundgesamtheit der Technologiebranche um neue Geschäftsjahre erweitert werden. Zum anderen können die Grundgesamtheiten von kleineren Branchen durch zusätzliche Geschäftsjahre so groß werden, dass abschlusspolitische Ergebnisspaltungen untersucht werden können. Bei diesen beiden Erweiterungen könnten vor allem die folgenden Fragestellungen berücksichtigt werden:

- In der vorliegenden Arbeit kann das Verschleiern von ineffizienten Unternehmensgrößen als Motiv für abschlusspolitische Ergebnisspaltungen in der Technologiebranche belegt werden. Lässt sich dieses Motiv auch in größeren Stichproben sowie in anderen Branchen feststellen?

- In der vorliegenden Arbeit können abschlusspolitische Ergebnisspaltungen beim Erhöhen der Ergebnisses aus den fortgeführten Geschäftsbereichen und abschlusspolitischen Ergebnisspaltungen beim Erreichen von Referenzwerten nur mit einer alternativen Berechnung der unerwarteten Differenzen der bereinigten Betriebsergebnismargen belegt werden, die empirisch aber sogar einfacher als das bisherige Verfahren ist. Lassen sich abschlusspolitische Ergebnisspaltungen beim Erhöhen der Ergebnisse aus den fortgeführten Geschäftsbereichen und beim Erreichen von Referenzwerten mit der Alternativmethode auch in größeren Stichproben sowie in anderen Branchen nachweisen?

- Insolvenzen sind die deutlichsten Zeichen für Marktaustritte von Unternehmen mit ineffizienten Größen. Allerdings treten Insolvenzen bei börsennotierten Unternehmen zu

1703 Böcking, H.-J./Worret, D. (2016), S. 123-126 identifizieren in zehn durch die DPR veranlassten Fehlerbekanntmachungen Verstöße gegen den IFRS 5. 
selten auf, um sie in der vorliegenden Arbeit untersuchen zu können. Lassen sich in größeren Stichproben abschlusspolitische Ergebnisspaltungen bei Unternehmen, die später insolvent werden, feststellen?

- In der vorliegenden Arbeit werden die Wirkungen von internen Corporate-GovernanceMechanismen und Qualitätsmerkmalen der Abschlussprüfungen auf abschlusspolitische Ergebnisspaltungen separat untersucht. Verstärken sich diese potenziellen Präventivmaßnahmen bei abschlusspolitischen Ergebnisspaltungen gegenseitig?

- In der vorliegenden Arbeit wird zwar die Existenz von abschlusspolitischen Ergebnisspaltungen festgestellt, aber nicht untersucht, ob Abschlussadressaten durch abschlusspolitische Ergebnisspaltungen in die Irre geführt werden. ${ }^{1704} \mathrm{Ob}$ Abschlussadressaten die verzerrten Informationsgrundlagen erkennen, könnte anhand der Wertrelevanz der Ergebnisse aus den aufgegebenen Geschäftsbereichen untersucht werden. ${ }^{1705}$ Falls die von abschlusspolitischen Ergebnisspaltungen betroffenen Ergebnisse aus den fortgeführten Geschäftsbereichen weniger wertrelevant sind als diejenigen Ergebnisse aus den fortgeführten Geschäftsbereichen, die nicht von abschlusspolitischen Ergebnisspaltungen betroffen sind, scheinen zumindest die Aktionäre abschlusspolitische Ergebnisspaltungen zu erkennen. ${ }^{1706}$ Falls die Wertrelevanz der Ergebnisse aus den fortgeführten Geschäftsbereichen dagegen nicht von abschlusspolitischen Ergebnisspaltungen abhängen, scheinen die Aktionäre diese zu übersehen. Zumindest für den US-amerikanischen Rechtsraum können Alfonso/Cheng/Pan (2015) zeigen, dass Investoren die Core Earnings bei abschlusspolitischen Ergebnisspaltungen überbewerten. ${ }^{1707}$

Ferner existieren bezüglich aufgegebenen Geschäftsbereichen Fragestellungen, die in der vorliegenden Arbeit weder theoretisch noch empirisch beleuchtet werden, aber dennoch von Interesse sein können:

- Können andere Theorien als die Transaktionskostentheorie, die Prinzipal-AgentenTheorie und Prospect Theory die Existenz und Prävention von abschlusspolitischen Ergebnisspaltungen erklären? Wie im Abschnitt 11.6 angesprochen wurde, können diese drei Theorien die Existenz und Prävention von abschlusspolitischen Ergebnisspaltungen nur sehr eingeschränkt erklären. ${ }^{1708}$

1704 Vgl. Chagnaadorj, O. (2018), S. 76. Für zur Veräußerung gehaltene langfristige Vermögenswerte vermuten Rogler, S./Tettenborn, M./Straub, S. V. (2012), S. 387 eine unzureichende Entscheidungsnützlichkeit allerdings nur dann, wenn diese Vermögenswerte keine aufgegebenen Geschäftsbereiche mit separat ausgewiesenem Ergebnisbeiträgen bilden.

1705 Analog zur Wertrelevanz von Anpassungen der GuV durch Vorstände Choi, Y.-S. et al. (2007), S. 618 f.

1706 Allerdings verursachen, wie im Abschnitt 1.2 ausgeführt wurde, abschlusspolitische Ergebnisspaltungen auch dann Effizienzeinbußen, wenn sie von den Abschlussadressaten erkannt werden.

1707 Siehe Alfonso, E./Cheng, C. S. A./Pan, S. (2015), S. 24 f.

1708 Allgemein zu konkurrierenden Theorien aus wissenschaftstheoretischer Sicht Atteslander, P. (2010), S. 26; Friedrichs, J. (1990), S. 72 f.; Hager, W. (1992), S. 8. 
- Werden beim erstmaligen Ausweis von aufgegebenen Geschäftsbereichen die Vorjahreszahlen abschlusspolitisch angepasst? Vorstände könnten den aufgegebenen Geschäftsbereichen in den Vorjahren niedrige und in den aktuellen Geschäftsjahren hohe Aufwendungen aus den fortgeführten Geschäftsbereichen abschlusspolitisch zuordnen, um den Abschlussadressaten steigende Ergebnisse aus den fortgeführten Geschäftsbereichen zu suggerieren.

- Sind abschlusspolitische Ergebnisspaltungen typischerweise nicht nachweisbar, da Vorstände den Ausweis von aufgegebenen Geschäftsbereichen abschlusspolitisch vermeiden? ${ }^{1709}$ Zum einen könnten Vorstände den Ausweis von aufgegebenen Geschäftsbereichen abschlusspolitisch vermeiden, falls sie den damit verbundenen administrativen Aufwand scheuen oder den Wettbewerbern die erforderlichen Erläuterungen zu den aufgegebenen Geschäftsbereiche vorenthalten wollen. ${ }^{1710}$ Zum anderen könnten Vorstände speziell den Ausweis von profitablen aufgegebenen Geschäftsbereichen abschlusspolitisch vermeiden, damit die positiven Ergebnisbeiträge im Ergebnis aus den fortgeführten Geschäftsbereichen verbleiben. ${ }^{1711}$ Im Gegensatz zum abschlusspolitischen Ausweis von aufgegebenen Geschäftsbereichen, der in der ergänzenden Analyse (2) analysiert wurde, lässt sich der abschlusspolitische Verzicht auf den Ausweis von aufgegebenen Geschäftsbereichen mit unternehmensexternen Daten aber kaum untersuchen. Vielmehr müsste anhand von unternehmensinternen Unterlagen beurteilt werden, ob die Definitionen von aufgegebenen Geschäftsbereichs erfüllt sind. ${ }^{1712}$

- Finden abschlusspolitische Ergebnisspaltungen auch abseits von aufgegebenen Geschäftsbereichen statt? Beispielsweise könnten Vorstände mehrdeutige Aufwandsarten bevorzugt unter den Vertriebs- oder Forschungskosten anstatt unter den Verwaltungskosten ausweisen, da sich die Abschlussadressaten aus Werbung oder Wissen möglicherweise höhere Wertschöpfungspotenziale erhoffen als aus bürokratischen Strukturen.

Abschließend sei angemerkt, dass das IASB nach jetzigem Stand keine wesentliche Überarbeitung des IFRS 5 plant, ${ }^{1713}$ wodurch die Rahmenbedingungen für empirische Untersuchungen von aufgegebenen Geschäftsbereichen zunächst stabil und die damit gewonnenen Erkenntnisse relevant bleiben. ${ }^{1714}$ Vor diesem Hintergrund wären weitere empirische

1709 Vgl. Gusinde, P. (2000), S. 235 f./240 f.

1710 Hierzu Coutinho e Silva, A. H. et al. (2018), S. 33; Kotowitz, J./Rhodes, C./Vanosdell, J. (2005), S. $37-$ 39; Schlaak, W. (2014), S. 232 f.

1711 Dazu Böcking, H.-J./Worret, D. (2016), S. 123; Rogler, S./Tettenborn, M./Straub, S. V. (2012), S. 384.

1712 Vgl. Rapaccioli, D./Schiff, A. (1991), S. 56.

1713 Selbst vom Exposure Draft „General Presentation and Disclosures“ (ED/2019/7) wurden aufgegebene Geschäftsbereiche gemäß ED/2019/7.BC13 Buchst. (a) explizit ausgenommen, obwohl ED/2019/7.4472 die Ergebnisspaltung in der GuV ausführlich thematisiert.

1714 Vgl. Keitz, I. v./Heyd, R. (2017), IFRS 5, Tz. 9; KPMG (Hrsg.) (2019), Tz. 5.4. Dagegen vermuten Böcking, H.-J./Kiefer, M. (2016), IFRS 5, Tz. 165; Nagengast, K./Boecker, C. (2017), S. 496 aufgrund der hohen Komplexität der Vorschriften und den zahlreichen Anwendungsfragen, dass IFRS 5 bald geändert wird. Allerdings hat sich diese Vermutung bislang nicht bestätigt. 
Arbeiten zur Rechnungslegung über aufgegebene Geschäftsbereiche im deutschen Rechtsraum ausdrücklich zu begrüßen.

\subsection{Zusammenfassung}

International Financial Reporting Standard 5 „Non-current Assets Held for Sale and Discontinued Operations“" verlangt, dass das Ergebnis aus den fortgeführten Geschäftsbereichen getrennt vom Ergebnis aus den aufgegebenen Geschäftsbereichen ausgewiesen wird. Abschlusspolitische Ergebnisspaltungen mit aufgegebenen Geschäftsbereichen sind $\mathrm{Zu}$ ordnungen von Aufwendungen bzw. Erträgen zu den fortgeführten oder aufgegebenen Geschäftsbereichen, bei denen Ermessensspielräume innerhalb des gegebenen Normenrahmens bewusst und zielgerichtet ausgenutzt werden. In der vorliegenden Arbeit wird untersucht, ob abschlusspolitische Ergebnisspaltungen mit aufgegebenen Geschäftsbereichen unter IFRS 5 existieren und ob sie verhindert werden können. Anhand von $325 \mathrm{Be}-$ obachtungen von CDAX-Technologieunternehmen aus den Jahren 2008 bis 2013 können abschlusspolitische Ergebnisspaltungen weder zum Erhöhen der Ergebnisse aus den fortgeführten Geschäftsbereichen noch zum Erreichen von Referenzwerten, zwecks Cookie Jars oder Big Baths belegt werden. Lediglich bei ineffizienten Unternehmensgrößen werden abschlusspolitische Ergebnisspaltungen festgestellt, die sich gegenüber diversen Modellmodifikationen als robust herausstellen. Die Prävention dieser abschlusspolitischen Ergebnisspaltungen kann nur bei hohen Verschuldungsgraden belegt werden. Dagegen können geeignete Aufsichtsräte, Großaktionäre, niedrige Prüfungshonorare abseits von externen Prüferrotationen und große Abschlussprüfer abschlusspolitische Ergebnisspaltungen bei ineffizienten Unternehmensgrößen nicht verhindern. Geeignete Vorstandsvergütungen, Nichtprüfungshonorare und Branchenspezialisierungen der Abschlussprüfer schwächen abschlusspolitische Ergebnisspaltungen bei ineffizienten Unternehmensgrößen zumindest $a b$.

Dieses Kapitel wird unter der Creative Commons Namensnennung 4.0 International Lizenz (http://creativecommons.org/licenses/by/4.0/deed.de) veröffentlicht.

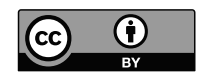

